\title{
Long term variability of gigahertz-peaked spectrum sources and candidates
}

\author{
I. Torniainen ${ }^{1}$, M. Tornikoski ${ }^{1}$, H. Teräsranta ${ }^{1}$, M. F. Aller ${ }^{2}$, and H. D. Aller ${ }^{2}$ \\ ${ }^{1}$ Metsähovi Radio Observatory, Helsinki University of Technology, Kylmäläntie 114, 02540 Kylmälä, Finland \\ e-mail: ilo@kurp.hut.fi \\ 2 Department of Astronomy, University of Michigan, Ann Arbor, MI, 48109 USA
}

Received 24 August 2004 / Accepted 14 February 2005

\begin{abstract}
We have combined new data from our observing campaigns and data from the literature to construct the radio continuum spectra for a sample of mostly quasar-type high peaking gigahertz-peaked spectrum (GPS) source candidates. We have also studied the spectra and variability of so called "bona fide" GPS sources and other inverted-spectrum sources from the literature. For many of our sample sources we now have data spanning over two decades, enabling us to study their long term behaviour.

Based on our earlier results we expected to find several new high peaking GPS sources. Instead we found out that even most of the "bona fide" GPS sources cease to adhere to the generic GPS source properties when using these well-sampled long term data sets.

In our sample of 35 inverted-spectrum sources from the literature only five seem to be consistent with the GPS properties, and even out of these sources two are too sparsely sampled to firmly make conclusions about their variability. Thirteen of the "bona fide" GPS sources exhibit pronounced activity, which diverges from the low variability expected from these sources. None of our new candidates turned out to have both a convex spectrum and little to no variability, but there is one variable source with a consistently convex spectrum. All the rest have flat spectra, but the upper envelope of the spectrum is clearly convex for four extremely variable sources. Similar continuum spectra with a flat lower envelope and a convex upper envelope are observed for eight previously identified inverted-spectrum sources.

According to this study the genuine quasar-type GPS sources are rare but there is a large number of highly variable sources that can have a convex spectrum peaking at high radio frequencies (up to ca. $100 \mathrm{GHz}$ ) during flares. Many of the GPS sources from the literature have too easily been classified as GPS sources based on too sparse data, and studying the long term variability is essential for identifying the sources with consistently convex continuum spectra.
\end{abstract}

Key words. galaxies: quasars: general

\section{Introduction}

Gigahertz-peaked spectrum (GPS) sources are extragalactic radio sources associated with either quasars or galaxies. Even though the characteristic convex spectral shape in the radio continuum peaking at the gigahertz frequencies is observed in both groups, the quasar-type GPS sources are often considered to be different from the galaxy-type GPS sources (e.g. Stanghellini et al. 1996; Snellen et al. 1998). In this paper we mainly concentrate on the sources associated with quasars.

Originally quasar-type GPS sources have been defined to have convex radio continuum spectra peaking at the frequency of approximately $1 \mathrm{GHz}$, low optical and radio polarization, rarely any variability at the radio frequencies and rarely superluminal motion (O'Dea 1998, and references therein).

Since the study of Rudnick and Jones (1982) the convex spectrum has been associated with low variability and low polarization. They compiled the broadband spectra for 40 flat-spectrum sources using data from Kühr et al. (1981) and presumed the data to be "sufficient for a crude characterization of the spectral shapes of individual sources" (Rudnick \& Jones 1982). Indeed, until now the GPS sources and the possibly related compact steep spectrum (CSS) sources are considered to be the least variable classes of extragalactic radio sources (O’Dea 1998).

Variability of GPS sources has been studied by Jauncey et al. (2003). They found different kinds of variability behaviour among southern hemisphere GPS sources, both quasars and galaxies. They found that $\sim 10 \%$ of the sources vary but they did not define exactly how active a source must be to be considered variable. They however mention a source to "exhibit strong variability" when it doubled its flux density in a year. They mention GPS sources that vary and also sources whose GPS spectrum is ephemeral on a timescales of years.

De Vries et al. (1997) studied the spectral shape of a GPS source sample including both quasars and galaxies. They included only sources with observed turnover frequency $\left(v_{\mathrm{T}}\right)$ 
between 0.5 and $10 \mathrm{GHz}$. They constructed a canonical GPS radio spectrum using data from the literature. The spectrum has spectral indices ${ }^{1}(+0.51 \pm 0.03)$ for the inverted part, $(-0.36 \pm$ $0.05)$ for the flat part following the peak and $(-0.73 \pm 0.06)$ for the optically thin part.

Based on their studies of optical spectroscopy and redshifts of GPS quasars and galaxies, Snellen et al. (1999) suggested that the GPS quasars are related to flat-spectrum radio sources rather than the GPS galaxies. They found that the optical spectra of quasar-type GPS sources is indistinguishable from the spectra of flat-spectrum quasars and differs from the spectra of CSS quasars.

There are several scenarios for the intrinsic nature of the GPS sources. One claims that they could be young precursors of the CSS sources and the extended radio sources (e.g. Mutel \& Phillips 1988; Fanti et al. 1990, 1995). Another suggests that the small size and the inverted spectrum are caused by dense environment that prohibits the source from growing bigger (e.g. Gopal-Krishna \& Wiita 1991). A third one, the so-called recurrent activity scenario, is based on some observations which have revealed faint extended emission around GPS sources and suggests that the central engine has on and off periods of activity (e.g. Baum et al. 1990).

Lately there has been evidence for GPS sources with turnover at high radio frequencies far above the classical $1 \mathrm{GHz}$ (e.g. Edge et al. 1996, 1998; Grainge \& Edge 1999; Tornikoski et al. 2001). Dallacasa et al. (2000) presented a sample of sources with inverted spectra peaking above $5 \mathrm{GHz}$ and named them high frequency peakers (HFP). After studying the sizes of the sources they suggested that these HFPs might represent an early phase of GPS-CSS-extended radio source evolution. They also noted that flux density variability is common among the HFP sources.

The study of the high radio frequency behavior of extragalactic sources is important for ESA's Planck Surveyor mission $^{2}$ that will be launched in 2007. The knowledge of the nature, number and behaviour of foreground sources is essential for the success of the Planck primary mission which is the measurement of the cosmic microwave background (CMB) radiation (Toffolatti et al. 1997). Our group has been especially interested in the extreme-peaking GPS sources. If such a source population exists, they may have been excluded from earlier high-frequency radio studies because most of the source samples for millimetre-domain studies have been selected using historical low-frequency data, assuming that sources that are bright enough to be detected in the mm-domain should be very bright in the low-frequency domain.

Our earlier work (Tornikoski et al. 2000, 2001, 2002) has indeed shown that there are several objects which have relatively faint historical low-frequency fluxes, but which can become bright in the mm-domain at least in the active state. If these sources are true GPS-type sources with convex spectra at all times, they should be detectable at the Planck frequencies up to ca. 200-300 GHz whenever they are in the Planck field of view. The knowledge of the existence of such objects

\footnotetext{
${ }^{1} S \propto v^{\alpha}$.

2 http://www.rssd.esa.int/Planck
}

is important for the understanding of the Planck foreground, especially because the continuum spectrum of such extremepeaking sources can resemble the shape of the CMB spectrum. On the other hand, if these sources turn out not to be "genuine" GPS sources but instead objects that have inverted spectra only during the active state, understanding their variability behaviour is important for estimating their impact (brightnesses and duty cycles) on the foreground emission at the Planck frequencies.

Thus we wanted to study the long-term variability of the "bona fide" GPS sources from the literature, and to identify new inverted-spectrum sources, especially ones peaking at very high radio frequencies and thus being of special interest for the work being done on the extragalactic foreground of the Planck satellite.

In Sect. 2 we describe the selection of the source sample, the observing methods and the data gathered from the literature. In Sect. 4 we present the results, the radio spectra and the interpretations for all the sources in the sample. In Sect. 5 we discuss the results and their implications.

\section{Sample and observations}

The sample used in this study was originally selected among the sources in the Metsähovi monitoring programme of extragalactic radio sources (Salonen et al. 1987; Teräsranta et al. 1992, 1998) and the monitoring programme of southern sources with the SEST-telescope (Tornikoski et al. 1996, 2005 , in preparation for A\&A). First the radio continuum spectra of the sources were plotted and the most promising new inverted-spectrum source candidates were selected for further study. Also all the known GPS sources found in the literature and included in the monitoring programmes were selected. Some sources were included in 2001 so only relatively shortterm variations could be studied.

The sample consists of 60 sources listed in Tables 1 and 2. Most of the sources are quasars or blazars. Some of the sources have been intensively monitored for many years and show pronounced variability at high radio frequencies. Some of the sources also only have few high-frequency data points because they were excluded from the early monitoring programs after they were considered to be uninteresting. These sources were selected and especially prioritized in our observation programme.

The observations for this paper were made in Finland with the $14-\mathrm{m}$ diameter Metsähovi radio telescope $\left(24^{\circ} 23^{\prime} 38^{\prime \prime} \mathrm{E}\right.$, $\left.+60^{\circ} 13^{\prime} 05^{\prime \prime}\right)$ at the frequency of $37 \mathrm{GHz}$. The observation programme started in November 2001 and is still going on. The latest data included in this paper were observed in January 2004. Thus we have monitored the variability of these invertedspectrum sources for more than two years.

The observations and the data reduction were made as described in Teräsranta et al. (1998).

For this study we have also had observing time at the Swedish-ESO Submillimetre Telescope (SEST) at La Silla, Chile, which is a $15-\mathrm{m}$ diameter radio telescope at coordinates $70^{\circ} 44^{\prime} 04^{\prime \prime},-29^{\circ} 15^{\prime} 34^{\prime \prime}$. The observation runs were 24-28 Aug. 2001, 22-28 Aug. 2002, 20-21 Dec. 2002, 14-17 Feb. 2003, 1-3 Apr. 2003 and 1-3 Jun. 2003. However, 
Table 1. Previously identified GPS sources.

\begin{tabular}{|c|c|c|c|c|c|c|c|c|c|c|}
\hline $\begin{array}{l}\text { Source } \\
\text { B1950 }\end{array}$ & ID & $z$ & $\begin{array}{l}\text { Original } \\
\text { class }\end{array}$ & Ref. & $\begin{array}{l}v_{\text {peak }} \\
\mathrm{GHz}\end{array}$ & $\alpha_{\text {below }}$ & $\alpha_{\text {above }}$ & $\begin{array}{l}\operatorname{Var}_{\Delta S, \max } \\
\max \end{array}$ & $\begin{array}{l}v_{\operatorname{Var}_{\Delta S} \max } \\
\mathrm{GHz}\end{array}$ & $\begin{array}{l}\text { Proposed } \\
\text { class }\end{array}$ \\
\hline 0048-097 & BLO & $\geq 0.2$ & GPS & 9 & 20.4 & 0.09 & -0.22 & 7.59 & 8 & $\mathrm{idb}$ \\
\hline $0153+744$ & LPQ & 2.338 & GPS & 4 & 2.1 & 0.70 & -0.76 & 5.33 & 37 & $\mathrm{c}$ \\
\hline $0215+015$ & BLO & 1.72 & HFP & 1 & 12.4 & 0.40 & -0.40 & 24.33 & 14.5 & $\mathrm{idb}$ \\
\hline $0218+357$ & BLO & 0.936 & GPS & 4 & $\ldots$ & $\ldots$ & -0.16 & 1.71 & 8 & $\mathrm{f}$ \\
\hline $0238-084$ & GAL & 0.0049 & GPS & 2 & $\ldots$ & $\ldots$ & -0.02 & 6.78 & 37 & $\mathrm{idb}$ \\
\hline $0248+430$ & LPQ & 1.316 & GPS & 5 & 4.9 & 0.28 & -0.40 & 6.95 & 37 & $\mathrm{f}$ \\
\hline $0252-549$ & QSO & 0.539 & inv & 9 & 37.4 & 0.51 & -0.89 & 0.76 & 90 & $\mathrm{c}$ \\
\hline $0332-403$ & HPQ & 1.445 & GPS & 9 & 10.6 & 0.17 & -0.17 & 1.69 & 90 & f \\
\hline $0402-362$ & LPQ & 1.417 & inv & 9 & 46.8 & 0.22 & -0.40 & 3.83 & 90 & $\mathrm{f}$ \\
\hline 0454-234 & HPQ & 1.003 & GPS & 9 & 9.2 & 0.10 & -0.28 & 3.05 & 90 & $\mathrm{f}$ \\
\hline $0528+134$ & BLO & 2.060 & GPS & 4 & 27.8 & 0.30 & -0.31 & 13.09 & 8 & $\mathrm{idb}$ \\
\hline 0537-441 & BLO & 0.896 & GPS & 9 & $\ldots$ & $\ldots$ & -0.01 & 4.33 & 90 & $\mathrm{f}$ \\
\hline 0539-057 & HPQ & 0.839 & inv & 9 & 5.8 & 0.20 & -0.31 & 0.66 & 37 & $\mathrm{f}$ \\
\hline $0552+398$ & QSO & 2.36 & GPS & 4 & 8.9 & 1.46 & -0.39 & 3.09 & 37 & $\mathrm{c}$ \\
\hline $0642+449$ & LPQ & 3.4 & HFP & 1 & 18.6 & 0.61 & -0.51 & 4.35 & 90 & $\mathrm{c}$ \\
\hline $0738+313$ & QSO & 0.631 & GPS & 4 & 2.6 & 0.26 & -0.30 & 2.94 & 37 & $\mathrm{f}$ \\
\hline $0741-063$ & N/A & N/A & GPS & 3 & 0.6 & 0.83 & -0.86 & 2.17 & 37 & gps \\
\hline $0742+103$ & GAL & 2.624 & GPS & 4 & 2.7 & 0.55 & -0.53 & 1.27 & 8 & gps \\
\hline 0743-006 & LPQ & 0.994 & GPS & 10 & 7.9 & 0.62 & -0.44 & 1.05 & 90 & gps \\
\hline $0906+430$ & HPQ & 0.668 & CSS & 11 & $\ldots$ & $\ldots$ & -0.77 & 3.61 & 37 & $\mathrm{~s}$ \\
\hline $0923+392$ & QSO & 0.698 & HFP & 1 & 11.0 & 0.49 & -0.32 & 7.33 & 8 & c \\
\hline $1057-797$ & QSO & N/A & GPS & 9 & 17.6 & 0.32 & -0.43 & 2.38 & 90 & $\mathrm{f}$ \\
\hline $1145-071$ & QSO & 1.342 & inv & 9 & 3.7 & 0.24 & -0.34 & 0.62 & 90 & $\mathrm{f}$ \\
\hline $1334-127$ & HPQ & 0.54 & GPS & 9 & 37.0 & 0.33 & -0.54 & 3.64 & 14.5 & $\mathrm{c}$ \\
\hline 1349-439 & BLO & 0.05 & GPS & 9 & 10.9 & 0.32 & -0.21 & 3.03 & 90 & $\mathrm{f}$ \\
\hline $1546+027$ & HPQ & 0.413 & inv & 9 & 35.6 & 0.18 & -0.17 & 4.16 & 90 & $\mathrm{f}$ \\
\hline $1637+574$ & LPQ & 0.75 & conv & 6 & $\ldots$ & $\ldots$ & -0.03 & 5.62 & 37 & $\mathrm{f}$ \\
\hline $1749+096$ & BLO & 0.32 & HFP & 1 & 37.1 & 0.36 & -0.60 & 18.16 & 90 & $\mathrm{idb}$ \\
\hline $1828+487$ & LPQ & 0.692 & CSS & 7 & $\ldots$ & $\ldots$ & -0.7 & 2.37 & 90 & $\mathrm{~s}$ \\
\hline $1936-155$ & HPQ & 1.657 & GPS & 8 & 11.9 & 0.21 & -0.23 & 4.58 & 90 & $\mathrm{f}$ \\
\hline 1954-388 & HPQ & 0.63 & GPS & 9 & 22.1 & 0.29 & -0.30 & 2.81 & 90 & $\mathrm{f}$ \\
\hline $2008-159$ & LPQ & 1.181 & GPS & 8 & 17.3 & 0.45 & -0.49 & 4.00 & 90 & $\mathrm{c}$ \\
\hline $2021+614$ & GAL & 0.2266 & GPS & 4 & 5.66 & 0.96 & -0.39 & 2.00 & 37 & gps \\
\hline $2022+171$ & LPQ & 1.05 & HFP & 1 & 17.8 & 0.44 & -0.77 & 0.41 & 90 & c \\
\hline $2121+053$ & QSO & 1.878 & HFP & 1 & 6.0 & 0.21 & -0.03 & 11.35 & 22 & $\mathrm{idb}$ \\
\hline $2128-123$ & LPQ & 0.501 & GPS & 9 & 9.9 & 0.24 & -0.53 & 1.03 & 90 & $\mathrm{c}$ \\
\hline $2134+004$ & LPQ & 1.932 & GPS,HFP & 5,1 & 4.9 & 1.1 & -0.51 & 2.62 & 90 & gps \\
\hline $2136+141$ & LPQ & 2.427 & GPS & 9 & 10.2 & 0.33 & -0.39 & 2.49 & 8 & f \\
\hline $2145+067$ & LPQ & 0.99 & inv & 9 & 21.9 & 0.39 & -0.39 & 6.16 & 22 & $\mathrm{idb}$ \\
\hline $2216-038$ & LPQ & 0.901 & inv & 9 & 7.4 & 0.30 & -0.72 & 3.34 & $\approx 5$ & $\mathrm{c}$ \\
\hline $2230+114$ & HPQ & 1.037 & CSS & 7 & $\ldots$ & $\ldots$ & -0.22 & 5.06 & 90 & $\mathrm{f}$ \\
\hline $2243-123$ & HPQ & 0.63 & inv & 9 & 8.8 & 0.12 & -0.17 & 1.77 & 90 & $\mathrm{f}$ \\
\hline 2254-204 & BLO & $\mathrm{N} / \mathrm{A}$ & GPS & 9 & 27.9 & 0.41 & -0.92 & 1.71 & 90 & $\mathrm{c}$ \\
\hline $2255-282$ & LPQ & 0.926 & GPS & 9 & 28.1 & 0.74 & -0.47 & 7.21 & 90 & $\mathrm{idb}$ \\
\hline
\end{tabular}

we have obtained only little data due to unfavorable weather conditions.

The $3 \mathrm{~mm}$ observations were made using a spectral line receiver centered at $90 \mathrm{GHz}$. The measurements were made with a $1 \mathrm{GHz}$-band SIS receiver. The SIS receiver was only used in a single-sideband (SSB) mode. As a backend, a wide band $(1 \mathrm{GHz})$ acousto-optic spectrometer (AOS) was used. The receiver temperature for the SIS receiver was typically $T=100-130 \mathrm{~K}$. The $3 \mathrm{~mm}$ observing procedures, pointing checks and intensity calibrations are as described in Tornikoski et al. (1996). The June 2003 observations were made with the
SIMBA bolometer centered at $250 \mathrm{GHz}(1.2 \mathrm{~mm})$. For flux calibration Uranus was observed also in fast mapping mode, as the primary target, and opacity corrections were made using frequent skydip observations. The maps were reduced using the standard reduction package MOPSI by Robert Zylka from IRAM, Grenoble, France.

The University of Michigan variability program uses a 26-meter prime focus paraboloid equipped with transistor based radiometers operating at center frequencies of 4.8, 8.0, and $14.5 \mathrm{GHz}$; their bandwidths are 560, 760, and $1600 \mathrm{MHz}$, respectively. All three frequencies utilize rotating, dual-horn 
Table 2. Candidate GPS sources.

\begin{tabular}{lllllllll}
\hline \hline $\begin{array}{l}\text { Source } \\
\text { B1950 }\end{array}$ & ID & $z$ & $\begin{array}{l}v_{\text {peak }} \\
\mathrm{GHz}\end{array}$ & $\alpha_{\text {below }}$ & $\alpha_{\text {above }}$ & $\begin{array}{l}\operatorname{Var}_{\Delta S} \\
\max \end{array}$ & $\begin{array}{l}v_{\text {Var }} \max \\
\mathrm{GHz}\end{array}$ & $\begin{array}{l}\text { Proposed } \\
\text { class }\end{array}$ \\
\hline $0221+067$ & HPQ & 0.511 & $\ldots$ & $\ldots$ & -0.001 & 3.34 & 37 & $\mathrm{f}$ \\
$0306+102$ & BLO & 0.836 & 21.4 & 0.28 & -0.03 & 5.36 & 8 & $\mathrm{f}$ \\
$0316+413$ & GAL & 0.0172 & 5.0 & 0.08 & -0.36 & 8.09 & 37 & $\mathrm{f}$ \\
$0420-014$ & HPQ & 0.915 & 37.0 & 0.27 & -0.73 & 8.12 & 90 & $\mathrm{c}$ \\
$0748+126$ & LPQ & 0.880 & 37.0 & 0.11 & -1.0 & 2.03 & 37 & $\mathrm{f}$ \\
$0754+100$ & BLO & 0.266 & 11.3 & 0.23 & -0.30 & 7.91 & 37 & idb \\
$0823+033$ & HPQ & 0.506 & 21.6 & 0.13 & -0.05 & 2.91 & 4.8 & $\mathrm{f}$ \\
$0828+493$ & BLO & 0.548 & 5.4 & 0.03 & -0.02 & 5.47 & 8 & $\mathrm{f}$ \\
$0829+046$ & BLO & 0.18 & 12.9 & 0.19 & -0.28 & 6.2 & 8 & idb \\
$0953+254$ & LPQ & 0.712 & 9.4 & 0.22 & -0.10 & 3.13 & 8 & $\mathrm{f}$ \\
$1148-001$ & QSO & 1.983 & $\ldots$ & $\ldots$ & -0.26 & 2.35 & 90 & $\mathrm{f}$ \\
$1308+326$ & BLO & 0.996 & 13.8 & 0.22 & -0.30 & 8.25 & 22 & idb \\
$1642+690$ & HPQ & 0.751 & $\ldots$ & $\ldots$ & -0.11 & 5.54 & 8 & $\mathrm{f}$ \\
$1730-130$ & BLO & 0.902 & $\ldots$ & $\ldots$ & -0.03 & 4.86 & 14.5 & $\mathrm{f}$ \\
$1741-038$ & HPQ & 1.057 & $\ldots$ & 0.02 & $\ldots$ & 6.63 & 22 & idb \\
$2131-021$ & BLO & 1.285 & 2.3 & $\ldots$ & -0.16 & 7.85 & 14.5 & $\mathrm{f}$ \\
\hline
\end{tabular}

polarimeter feed systems, which permit both total flux and linear polarizations to be measured using an on-off observing technique at $4.8 \mathrm{GHz}$ and an on-on technique, (switching the target source between the two feed horns closely spaced on the sky), at the other two frequencies. A typical observation consists of 8 to 16 individual measurements over a 20 to 40 minute period. Frequent drift scans are made across stronger sources to verify the telescope pointing correction curves; and observations of program sources are intermixed with observations of a grid of calibrator sources: used to correct for temporal changes in the antenna aperture efficiency. The flux scale is set by observations of Cassiopeia A (e.g. see Baars et al. 1977). Details of the calibration and analysis techniques are described in Aller et al. (1985).

In addition to our own monitoring data we gathered data from the literature, the references are listed in Table 3.

In addition to plotting the continuum spectra, we have also calculated variability and spectral indices for the sources. Below we use the fractional variability index $\operatorname{Var}_{\Delta S}=\left(S_{\max }-S_{\min }\right) / S_{\min }$ at the given frequency. Since we are interested in the maximum amount of variability that a source can have and since our frequency coverage in the monitoring data is quite extensive, only the greatest value of the variability index and its frequency were extracted to Tables 1 and 2 .

In order to get the spectral indices and the peak frequencies we fitted the following function from Dallacasa et al. (2000) to the data:

$\log S=a-\sqrt{\left.b^{2}+(c \log v-d)^{2}\right)}$

It is a purely analytical function originating from the equation of Kovalev et al. (2000) rearranged by Dallacasa et al. (2000) with no physical meaning. The parameters are solely numeric and the main purpose of the function is only to model the spectral shape. This function was used to derive the turnover frequency $\gamma_{\text {peak }}$. The spectral indices $\alpha_{\text {below }}$ and $\alpha_{\text {above }}$ of the
Table 3. References for the data taken from the literature.

\begin{tabular}{ll}
\hline \hline Reference & Reference \\
\hline Beasley et al. (1997) & Owen et al. (1980) \\
Becker et al. (1991) & Pauliny-Toth et al. (1978) \\
Bondi et al. (1996) & Peng et al. (2000) \\
Dagkesamanskii et al. (2000) & Perley (1982) \\
Dallacasa et al. (2000) & Reich et al. (1998) \\
Douglas et al. (1996) & Reich et al. (2000) \\
Drinkwater et al. (1997) & Reuter et al. (1997) \\
Edelson et al. (1987) & Robson et al. (2001) \\
Gear et al. (1994) & Seielstad et al. (1983) \\
Geldzahler \& Witzel (1981) & Steppe et al. (1995) \\
Ghosh et al. (1994) & Teräsranta et al. (1987) \\
Gregory \& Condon (1991) & Teräsranta et al. (1992) \\
Gregory et al. (1994) & Teräsranta et al. (1998) \\
Griffith et al. (1995) & Tornikoski et al. (1993) \\
Kovalev et al. (1999) & Ulvestad et al. (1981) \\
Kühr et al. (1981) & Visser et al. (1995) \\
Large et al. (1991) & Wiren et al. (1992) \\
Lawrence et al. (1983) & Wright et al. (1991) \\
O’Dea et al. (1990) & Wright et al. (1996) \\
Verkhodanov et al. (1997, and the references therein) \\
\hline
\end{tabular}

overall spectrum were then derived by fitting a straight line separately to the parts below and above the peak frequency. For the flat or steep spectrum sources with $v_{\text {peak }}$ not within the data range only one line was fitted and there is only one spectral index describing the whole radio spectrum. For the convex sources B0552+398, B2021+614 and B2134+004 the data range for the fit of $\alpha_{\text {below }}$ was limited to frequencies well below the turnover frequency in order to get a spectral index that better describes the clearly inverted part of the spectrum and ignores the flattening near the turnover. For all the other sources with convex spectrum the fits were in good agreement with the spectral shape. 


\section{Source classification}

The sources in the sample were divided into five different categories (flat, steep, gps, convex, inverted during bursts) according to their spectral shape, spectral indices, turnover frequency and fractional variability indices. The shape of the spectrum examined visually was the most important criterion in classifying the sources but the parameters mentioned above were used to draw a line between the different classes for the intermediate cases. The sources with simply declining spectrum were divided according to the established spectral index division: sources with the overall spectral index less than -0.5 were classified as steep spectrum sources (s) and sources with the overall spectral index more than -0.5 and less than 0.5 were classified as flat spectrum sources (f). Also all the spectra with convex features, but both spectral indices in between -0.5 and 0.5 were labelled as flat. There is a significant share of previously identified GPS or HFP sources and candidates, which exhibit remarkable variability $\left(\operatorname{Var}_{\Delta S \max }>6\right)$ and the upper envelope of the overall spectrum is convex but the lower envelope rather flat or steep. These sources have inverted spectra during bursts (idb) but the turnover frequency and the shape of the spectrum vary with activity and the spectral shape can be flat or steep at quiescent times.

The sorting of the sources with convex spectrum was more complicated. First of all, it is difficult to distinguish between truly convex spectrum and undersampled flat-spectrum observed only in the state of high activity. Long term monitoring is needed to tell the difference and for some sources with data from only one epoch or sparse data with no date information, the identification is uncertain. In these cases we have labelled the spectra as convex even though more extensive monitoring can reveal the possible flat nature of the quiescent spectra. On the other hand, for the convex sources in our sample there is no clear division into variable and non-variable groups, so after visual examination we chose a somewhat arbitrary borderline of $\operatorname{Var}_{\Delta S \max }=3$. Taking also into account the spectral index limit of $\alpha_{\text {below }}>0.5$ for inverted spectrum and the visually confirmed convex spectral shape, we labelled the sources with high activity $\left(\operatorname{Var}_{\Delta S \text { max }}>3\right.$ ) as convex sources to differentiate them from genuine GPS sources. The few sources with beautifully convex spectra $\left(\alpha_{\text {below }}>0.5\right)$ and low variability $\left(\operatorname{Var}_{\Delta S \max }<\right.$ 3 ) were classified as genuine GPS sources (gps). The classification also required that the shape of the spectrum does not leave much room for suspicion that undersampling would hide the flat nature of the lower envelope and for this reason some sources with correct parameters fell out of the gps into the convex category.

Notes on Tables 1 and 2:

Col. 1: B1950.0 name of the source. Sources which remain true GPS sources are indicated with boldface type;

Col. 2: optical identification. BLO = BL Lacertae object, $\mathrm{GAL}=$ galaxy, HPQ = high optical polarization quasar, LPQ = low optical polarization quasar, QSO = quasar, $\mathrm{N} / \mathrm{A}=$ identification not available;

Col. 3: Redshift, N/A = redshift not available;

Col. 4: (only in Table 1) original classification or description from the literature. GPS = gigahertz-peaked spectrum source, CSS = compact steep-spectrum source, HFP = high frequency peaker, inv = invertedspectrum source, conv = convex spectrum;

Col. 5: (only in Table 1) reference for Col. 4:

1 Dallacasa et al. (2000)

2 de Vries et al. (1997)

3 Jeyakumar et al. (2000)

4 O’Dea et al. (1991)

5 O'Dea et al. (1998)

6 Rudnick \& Jones (1982)

7 Spencer et al. (1989)

8 Tornikoski et al. (2000)

9 Tornikoski et al. (2001)

10 Stanghellini et al. (1998)

11 Fanti et al. (1985);

Col. 6 (Col. 4 in Table 2): frequency of the spectral turnover from the fit described above;

Col. 7 (Col. 5 in Table 2): spectral index below the turnover;

Col. 8 (Col. 6 in Table 2): spectral index above the turnover;

Col. 9 (Col. 7 in Table 2): the greatest value of the fractional variability index $\operatorname{Var}_{\Delta S}$ in the data range;

Col. 10 (Col. 8 in Table 2): frequency of the greatest variability index given in Col. 9;

Col. 11 (Col. 9 in Table 2): classification of the spectra proposed in this paper. gps = genuine GPS source, $\mathrm{c}=$ convex, $\mathrm{s}=$ steep, $\mathrm{idb}=$ inverted during bursts, $\mathrm{f}=$ flat.

\section{Results}

\subsection{Source sample}

Our sample consisted of 60 sources of which 21 were classified in the literature as a HFP, GPS or CSS source and one was mentioned to have a convex spectrum. Additionally, 14 sources have been identified as GPS sources and eight sources as inverted-spectrum sources earlier by our research group (Tornikoski et al. 2000, 2001). The rest 16 sources were selected because in the early phase of this study, when only parts of these data were available, their continuum spectra seemed to be inverted. Already then we realized that some of these spectral shapes are probably due to undersampled data, but because many of the GPS classifications in the literature have been done on the basis of relatively sparse data sets, we included these sources in this paper. We want to present also these plots as examples of cases where sparser but unbiased data sets could have placed them among suggested new GPS sources, and to show that there is an abundance of sources that can have inverted, extreme-peaking spectra during their activity states, even though they should not be classified as genuine GPS sources.

Morphologically 56 of the sources were quasars, three of the previously identified GPS sources and one GPS candidate were galaxies.

After studying the spectra, we found that there were only five sources still in agreement with classical GPS properties. This is only $8.3 \%$ of all the sources in the sample and $19.2 \%$ of the previously identified GPS sources. 


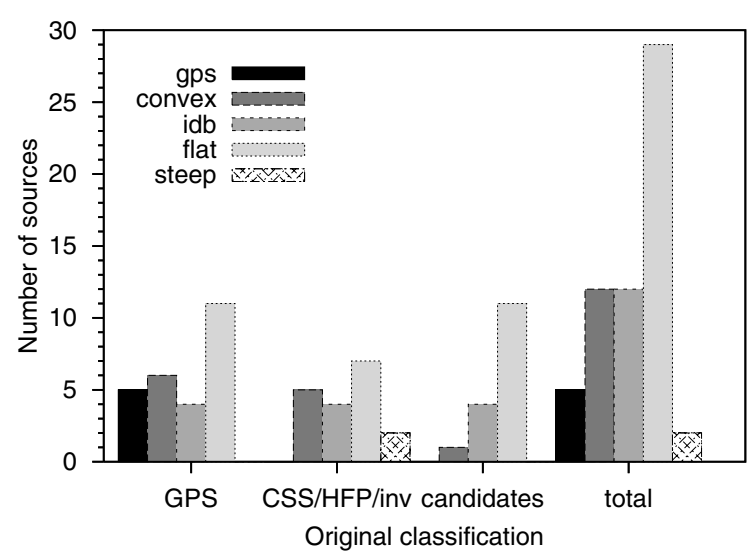

Fig. 1. Histogram showing our source classification segmented into the original categories.

Almost a half of all the sources (48.3\%) as well as of the GPS sources $(40.9 \%)$ had a flat spectrum. Twelve sources ( $\sim 20 \%$ of the overall sample) turned out to be variable flatspectrum sources with convex upper envelope and extremely high variability $\left(\operatorname{Var}_{\Delta S \text {, max }}>6\right)$, the proportion of them in the previously identified "bona fide" GPS sample is $15.4 \%$. Convex spectra and at least moderate variability $\left(\operatorname{Var}_{\Delta S, \max }>\right.$ 3 ) were observed among 12 sources (20\% of all and $23 \%$ of the "bona fide" sample). There is a histogram in Fig. 1 which shows how many sources of each new category are found among the original classes. Histograms of the greatest value of fractional variability index for original classification and our proposed classification are presented in Fig. 2. It clearly shows that variability is common among previously identified GPS sources while our genuine GPS sources are quite quiescent.

More detailed statistical analysis is left to the following paper (Torniainen et al. 2005, in preparation).

In the next two sections we present the radio continuum spectra of our sample. All the GPS, CSS and HFP sources from the literature are presented in Sect. 4.2 despite of our interpretation of their spectra. The candidate GPS sample is presented in Sect. 4.3.

Unless otherwise mentioned the redshift information has been taken from the NASA/IPAC Extragalactic Database (NED).

\subsection{Previously identified inverted-spectrum sources}

B0048-097: is a typical BL Lac object: it is highly polarized $\left(p_{\text {opt }}=10.6 \%\right.$ Wills et al. 1992), bright, variable and its line spectrum is featureless preventing the redshift determination (Gabuzda et al. 1999). However, a lower limit of $z>\approx 0.2$ was derived by Stickel et al. (1993) from the absence of the extended nebulosity of the host galaxy. In VLBI imaging Gabuzda et al. (1999) found the source to be very compact but there was evidence for a single jet. Also a secondary jet is possible, but greater resolution is required to ensure it.

This source was regocnized as an inverted-spectrum source with a turnover at $10 \mathrm{GHz}$ by Tornikoski et al. (2001). Simultaneous data from the end of 1992 from Hartebeesthoek Radio Astronomy Observatory (HartRAO) and Metsähovi
Radio Observatory show that the spectrum is inverted up to $22 \mathrm{GHz}$ when activity is medium. There is a significant amount of activity especially at frequencies above $5 \mathrm{GHz}$, the lower envelope of the spectrum is flat and the upper is clearly convex.

B0153+744: this quasar has a redshift of 2.338 (Peng et al. 2000). Hummel et al. (1988) imaged the source with VLBI. They found a single-sided strongly bent jet, which lead them to think that the ejection direction from the core has been changing. On the ground of the estimated jet speeds and rate of the change in the direction they calculated it to be a young source likely only less than 1000 years of age. The optical polarization of this source is $1.72 \%$ (Wills et al. 1992).

This source was labelled as a GPS source by O'Dea et al. (1991). Figure 3 shows a convex spectrum with only three data points of the optically thick part below $1 \mathrm{GHz}$ the rest of the spectrum is quite steep and variable. More data are needed to construct the lower frequency spectrum. There is variability at least at $22 \mathrm{GHz}$ and $37 \mathrm{GHz}\left(\operatorname{Var}_{\Delta S}(22)=2.96\right.$ $\operatorname{Var}_{\Delta S}(37)=5.33$ ) which is in contradiction with the classical GPS properties.

B0215+015: this source is a BL Lac object at redshift 1.72 (Falomo et al. 1994). It is observed to be a violently variable AGN (Schramm et al. 1994). Its optical polarization is high: $17.4 \%$ (Wills et al. 1992). Using simultaneous data (epoch 14 Jun., 1999) from the Very Large Array (VLA), which showed a convex spectral shape, Dallacasa et al. (2000) classified this source as a high frequency peaker (HFP) having a peak at a frequency of more than $22 \mathrm{GHz}$. Our plot in Fig. 3 confirms the convex shape of the upper envelope but the lower envelope is quite steep. Our data show that the source was having a flare at the time of Dallacasa et al. were observing it with the VLA in July, 1999. This source is indeed very variable $\left(\operatorname{Var}_{\Delta S}(37)=10.25\right)$ and during the outbursts the spectrum is inverted.

B0218+357: has been identified as a gravitational lens in the literature. The original source is at $z=0.936$ (Veron-Cetty $\&$ Veron 1998) and the lensing galaxy at $z=0.685$ (Browne et al. 1993). The lensed source is a BL Lac object (Stickel \& Kühr 1993), observed to be violently variable (Stickel et al. 1996).

The source was identified as a GPS candidate by O'Dea et al. (1991). Our plot does not show any evidence for the convexity. The spectrum declines slowly ( $\alpha=-0.16$ ) throughout the radio spectrum (Fig. 3), and even during the outbursts the spectrum seems to be only flat.

B0238-084: this source, also known as NGC 1052, is a bright elliptical Seyfert II-galaxy in the Cetus I cluster at a redshift of 0.0049 . It is known to have variability and an inverted radio spectrum (Condon et al. 1998). The source has been studied in detail by Vermeulen et al. (2003). They suggest that both synchrotron self-absorption and free-free absorption are present in the jets which are aligned close to the plane of the sky.

de Vries et al. (1997) mentioned this source to be a GPS source but our plot (Fig. 3) with new data shows a flat quiescent and a convex flaring spectrum.

B0248+430: the quasar has a redshift of $z=1.316$ (Small et al. 1997) and it is an LPQ with a polarization of $1.01 \%$ 

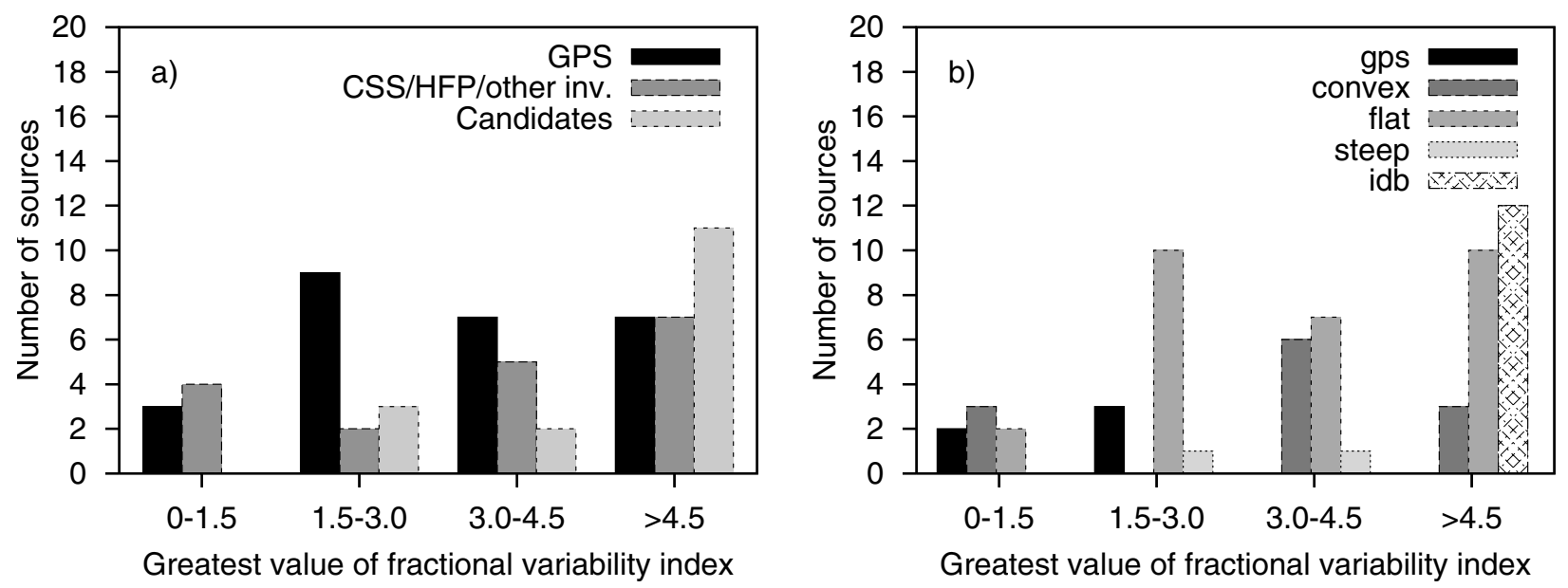

Fig. 2. Histograms of the maximum value of fractional variability index for a) the original classification from the literature and b) our classification.

(O’Dea et al. 1998). O’Dea (1998) listed this source as a GPS source from the Stanghellini et al. GPS source sample.

The spectrum in Fig. 3 has a mild convex shape but it can be only illusion created by the lack of variability observations at intermediate frequencies. More observations at frequencies from 1 to $10 \mathrm{GHz}$ are needed to decide whether the quiescent level is inverted or not. The variability at $1.4,22$ and $37 \mathrm{GHz}$ and the spectral indices however hint that in reality this is a variable flat-spectrum source.

B0252-549: this source is a quasar at $z=0.539$. Its spectrum seems to be convex with a turnover at $37 \mathrm{GHz}$ but the variability observed at $90 \mathrm{GHz}$ suggests that the lower envelope of the spectrum could be flat. The only data between 20 and $90 \mathrm{GHz}$ are Wilkinson Microwave Anisotropy Probe (WMAP) data from the CATS database (Verkhodanov et al. 1997) and there is no information on the simultaneous low frequency behaviour. Even though the parameters would qualify this source to be a genuine GPS source in our classification, the lack of data makes the interpretation of the spectra questionable and thus this source is classified as a convex-spectrum source.

B0332-403: this source is an HPQ $(z=1.445)$. It was identified as a GPS source by Tornikoski et al. (2001) but with all the new additional data the spectrum is rather flat.

B0402-362: this source is a low polarization quasar $(z=$ 1.417) The spectrum seems to be convex but the variability at 37 and $90 \mathrm{GHz}$ implies that the convexity is only apparent and due to too sparse data. The lower envelope of spectrum is flat and since the variability is only moderate $\left(\operatorname{Var}_{\Delta S, \max }=3.83\right)$, the source is labelled as flat.

B0454-234: this source is an HPQ at $z=1.003$. It was classified as a GPS source by Tornikoski et al. (2001) but the new dataset shows a declining spectrum inverted only during the outbursts. This source is also labelled as flat because the variability $\left(\operatorname{Var}_{\Delta S, \max }=3.05\right)$ is not high enough for the idb classification.

B0528+134: is also known as OG 147. It is one of the strongest $\gamma$-ray blazars and emits most of its power in the MeV energy range (Hunter et al. 1993). The redshift is 2.060 and there has been observed superluminal motion
(Peng et al. 2000). The latter implies that the angle of the jet is very close to the line of sight. The radio polarization of this source is quite high: $4.8 \%$ (Nartallo et al. 1998). O'Dea et al. (1991) identified this source as a GPS, but we find no evidence for the suggestion. The lower envelope of the spectrum (in Fig. 3) is very flat, remaining on the level of the low frequencies throughout the range covered by this study. The upper envelope shows a very clear inverted shape; thus this source is a flat-spectrum source with inverted spectrum during the outbursts. The same conclusion can be made from the simultaneous spectra from eight epochs by Kovalev et al. (2000). The greatest fractional variability index is 13.09 at $8 \mathrm{GHz}$.

B0537-441: this southern source was discovered to be a GPS source by Tornikoski et al. (2001). It lies at a redshift of 0.896 and is violently variable and active also in the $\gamma$-rays, also thought to be a gravitationally lensed object (Fan et Lin 1999 and the references therein). It is a transition object between BL Lac objects and quasars (Maraschi et al. 1985) and is morphologically a core-jet source but there has not been enough data to detect any proper motion in the jets (Shen et al. 1998).

The lower envelope of the spectrum in Fig. 3 is flat at low frequencies and steepens somewhere above $10 \mathrm{GHz}$. There is variability at high frequencies and the source is still bright at $352 \mathrm{GHz}$.

B0539-057: is a high polarization quasar $(z=0.839)$ identified to have an inverted spectrum by Tornikoski et al. (2001). However, the data from the CATS database (Verkhodanov et al. 1997) and the UMRAO at 5 and $8 \mathrm{GHz}$ make the spectrum flat and thus there is no evidence for a convex shape of the spectrum anymore.

B0552+398: this GPS source (O'Dea et al. 1991) is a bright quasar at $z=2.36$ (Hutchings 1992) with low polarization $\left(p_{\text {opt }}=2.34\right.$, Wills et al. 1992). It is also known as DA 193. Hutchings (1992) found a close companion 2.9" to the north connected to the quasar by tidal interactions. Both lie in a dense group of compact galaxies. Hutchings (1992) suggested that this source is young because it is compact, luminous, variable and gigahertz-peaked. Lister \& Marscher (1996) observed a short jet and possibly a counterjet, which can also be the core 

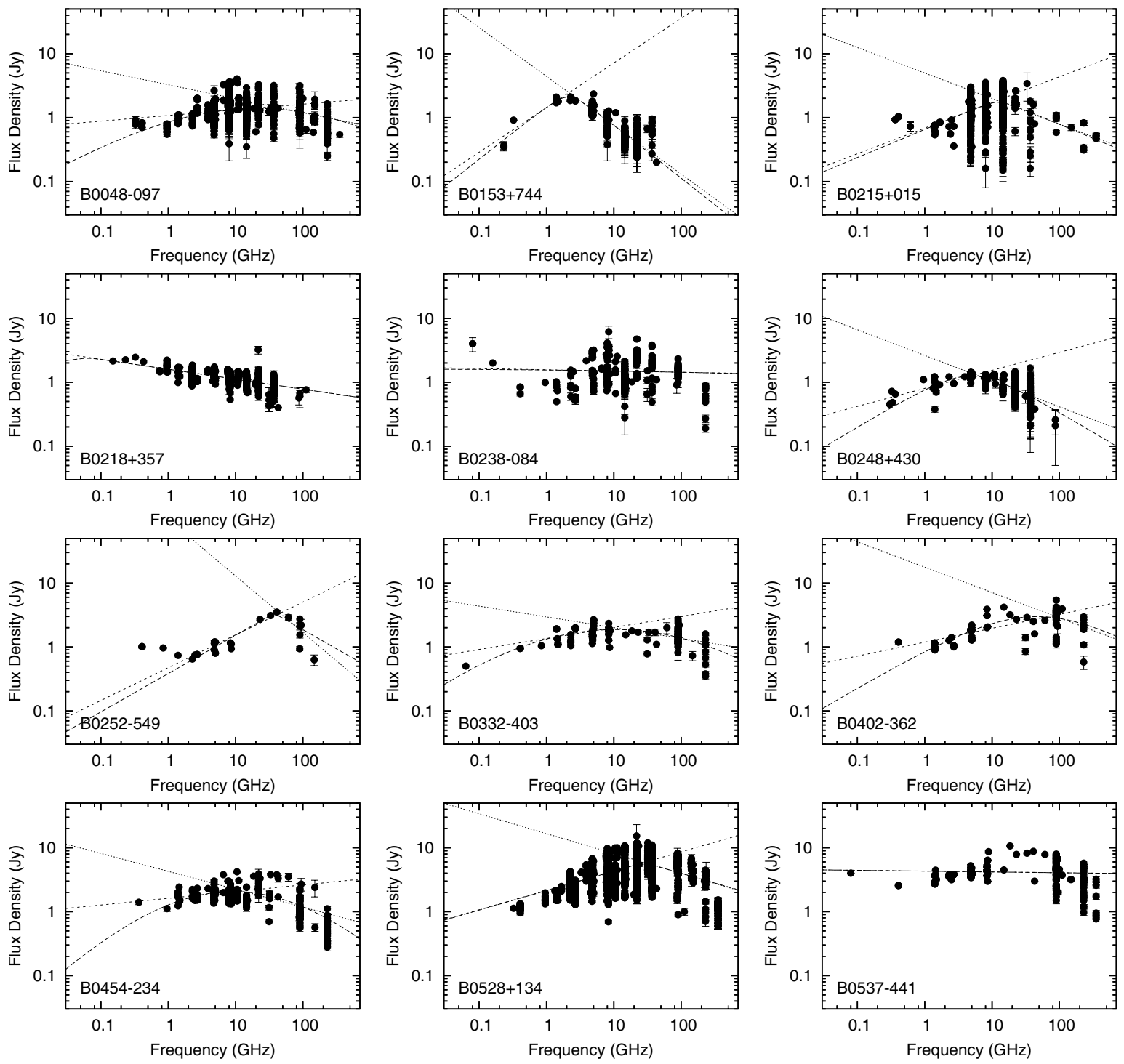

Fig. 3. Radio spectra of the previously identified inverted-spectrum sources.

of the source. They marked superluminal motion in the jet and possibly some signs of an extended component which would, if real, indicate a steep curve in the jet.

The shape in the plot (Fig. 4) is beautifully convex. The peak frequency of the minima is $\sim 5-8 \mathrm{GHz}$ and of the observed maxima $8-22 \mathrm{GHz}$. There is variability above the turnover $\left(\operatorname{Var}_{\Delta S}(37 \mathrm{GHz})=3.09\right)$ but the low frequency end of the spectrum seems to be quite steady, the $4.8 \mathrm{GHz}$ data contains observations from over ten years.

The spectral shape of this source is definitely convex, the inverted part has the greatest spectral index in the sample: 1.46. The variability is slightly too high to qualify this source to be a classical GPS by our criteria.

B0642+449: the redshift of this blazar is high: 3.4 (Donato et al. 2001). This source is a low-polarization quasar with a polarization of $1.67 \%$ (Wills et al. 1992). This source has been identified as a HFP by Dallacasa et al. (2000).

Our data in Fig. 4 show a clearly peaking spectral shape with the peak at $18.6 \mathrm{GHz}$. The spectral indices for the inverted and the steep part are +0.61 and -0.51 , respectively.
Variations are observed on the both sides of the turnover, especially at the high frequencies $\left(\operatorname{Var}_{\Delta S}(\sim 5 \mathrm{GHz})=3.19\right.$ and $\left.\operatorname{Var}_{\Delta S}(90 \mathrm{GHz})=4.35\right)$.

B0738+313: this is a known GPS quasar (O'Dea et al. 1991) with a redshift of 0.631 (Hewitt \& Burbidge 1993) and optical polarization of $0.32 \%$ (Wills et al. 1992). It is mentioned to have two close faint neighbours but it is not clear if they have any physical connections with the source (Hutchings 1992). The source itself has a jet with a well-defined sharp bend with a prominent knot at the bend (Kellermann et al. 1998).

The shape of the spectrum is slightly convex (Fig. 4) but the spectral indices are too flat for a convex source.

B0741-063: this source is relatively little studied; its redshift was not available in the NED or any other reference studied. It was labelled as a CSS source by Steppe et al. (1995) and later as a GPS by Jeyakumar et al. (2000).

The source is strong, over 10 Jy below $1 \mathrm{GHz}$ but declines steeply at higher frequencies. There is moderately variability at $37 \mathrm{GHz}\left(\operatorname{Var}_{\Delta S}(37 \mathrm{GHz})=1.85\right)$ The plot in Fig. 4 confirms the spectrum to be inverted with a peak at $600 \mathrm{MHz}$. 

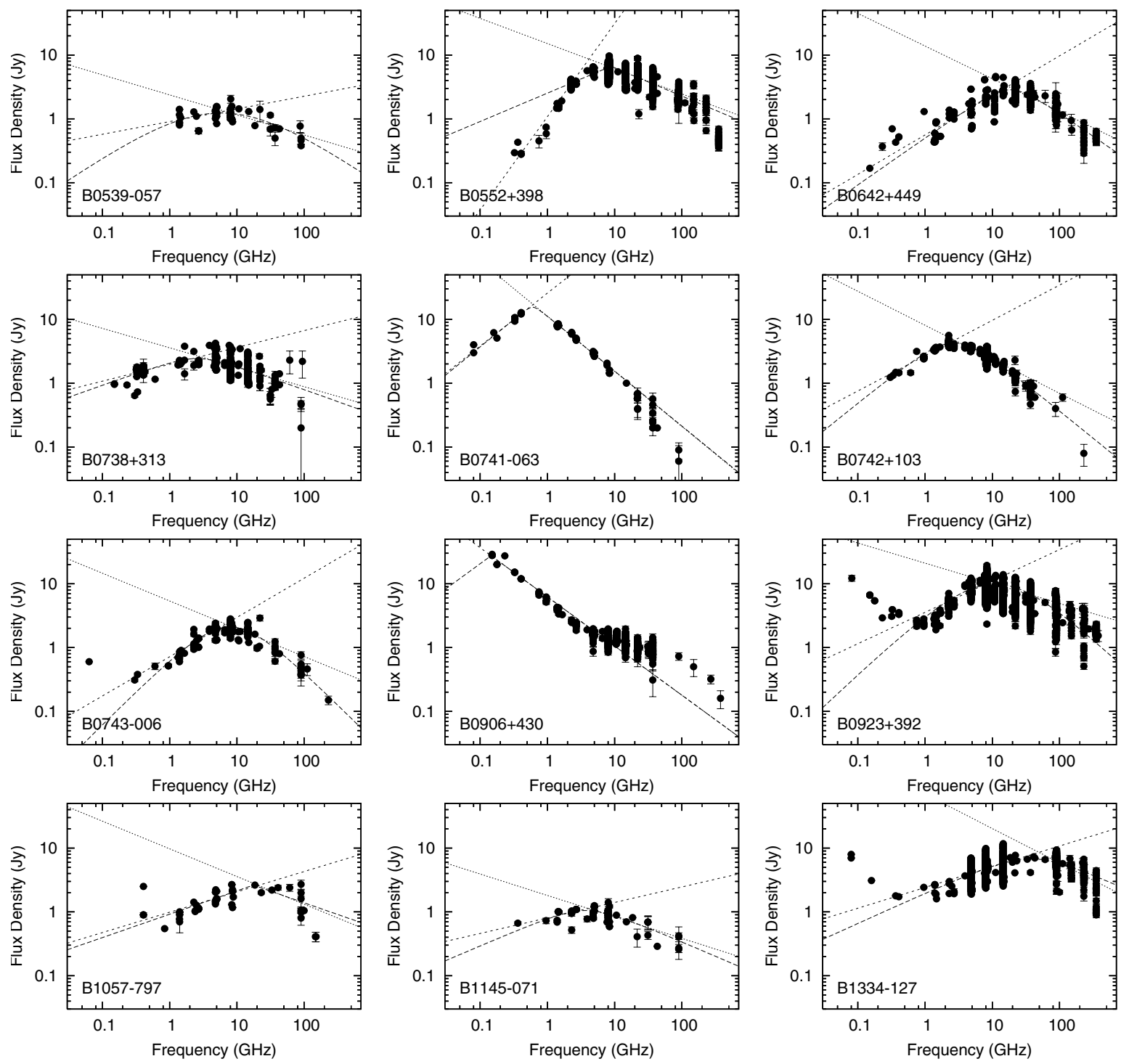

Fig. 4. Radio spectra of the previously identified inverted-spectrum sources.

However, there are at most two data points at each frequency below $10 \mathrm{GHz}$. With these data the source can be classified as a genuine GPS source.

B0742+103: this is a GPS galaxy identified by O'Dea et al. (1991). It is very bright in the radio but extremely weak in the optical (Stickel et al. 1996). The redshift of this source is high: $z=2.624$.

The spectrum in Fig. 4 is a smoothly curved GPS spectrum with a turnover at $2.7 \mathrm{GHz}$. The spectral index below the peak is +0.55 and above it -0.53 .

There are several observations at different epochs at a wide range of frequencies and still only little variability is observed. The variability index at $8 \mathrm{GHz}$ which is the best sampled frequency is 1.27 with a time span of almost 30 years. This is one of the rare remaining classical GPS sources in our study.

B0743-006: is a GPS quasar identified by Stanghellini et al. (1998) with a redshift of 0.994 (Hewitt \& Burbidge 1993 ) and polarization of $0.43 \%$ (Wills et al. 1992). The global VLBI observations at $5 \mathrm{GHz}$ have revealed only a very compact central component with a jet of a few milliarcseconds (Stanghellini et al. 1997).

Our data in Fig. 4 confirm the GPS shape in the spectrum. The turnover is at $7.9 \mathrm{GHz}$. The spectral indices are +0.62 and -0.44 for the optically thick and thin parts, respectively. There is quite little variability on both sides of the peak $\left(\operatorname{Var}_{\Delta} S(2.3 \mathrm{GHz})=1.05\right.$ and $\left.\operatorname{Var}_{\Delta S}(90 \mathrm{GHz})=1.05\right)$, still retaining the clear convex shape. This is another rare example of genuine GPS sources in this study.

B0906+430: this source, also known as 3C 216, was one of the first quasars discovered. It has properties of both the BL Lac objects and high polarization quasars (Villata et al. 1997) with an optical polarization of $3.34 \%$ (Wills et al. 1992). Its redshift is 0.668 . The source is a CSS source identified by Fanti et al. (1985). Barthel et al. (1988) found it to have superluminal motion. Liu \& Zhang (2002) report a jet $0.6 \mathrm{kpc}$ in projected length. However, Taylor et al. (1995) observed a diffuse dim halo around the source with a diameter of $40 \mathrm{kpc}$. They also claim that this source has clues for both the youth and the 
frustration scenarios, as well as the projection effect for the source's apparent smallness.

Our plot (Fig. 4) lacks the low frequency data which could show the peak below $\mathrm{MHz}$ frequencies. The rest of the spectrum is steep ( $\alpha=-0.77)$ and there is some variability at high frequencies $\left(\operatorname{Var}_{\Delta S}(37 \mathrm{GHz})=3.61\right)$.

B0923+392: this source is also known as 4C 39.25. It is a quasar $(z=0.698)$ with strong flux density variations. The radio polarization of this source is $5.6 \%$ (Nartallo et al. 1998).

Dallacasa et al. (2000) identified this source as a HFP. The spectrum in Fig. 4 has a convex shape peaking at $11 \mathrm{GHz}$. The spectral index below the peak is +0.49 and above -0.32 . There is significant variability at all frequencies (e.g. $\left.\operatorname{Var}_{\Delta S}(8 \mathrm{GHz})=7.33\right)$. Well below the peak the variability is less pronounced and at a time scale of five years during 1983-1988 the source was steady $\left(S_{2.7 \mathrm{GHz}} \approx 5 \mathrm{Jy}\right.$ and $S_{8.2 \mathrm{GHz}} \approx 5 \mathrm{Jy}-7 \mathrm{Jy}$, Waltman et al. 1991).

B1057-797: this is a very little studied source in the southern sky. The GPS classification was made by Tornikoski et al. (2001) and the current dataset does not disprove the convexity but there is moderate variability at $90 \mathrm{GHz}\left(\operatorname{Var}_{\Delta S}=2.38\right)$ that could imply that the shape of the spectrum is flat. The spectral indices are also too flat for a convex-spectrum source.

B1145-071: is a binary quasar with radio faint and radio bright components (Djorgovski et al. 1987) Its redshift is 1.342. The spectrum in Fig. 4 is rather flat with slightly convex upper envelope.

B1334-127: this is a GPS classified by Tornikoski et al. (2001). It has a redshift of 0.54 and is very luminous and compact source (Gabuzda et al. (2000). The source has a core and a jet that bends to the east $6.5^{\prime \prime}$ from the core (Lister \& Marscher 1996). Gabuzda et al. (2000) found the jet to be highly polarized $(18 \%)$ at $6 \mathrm{~cm}$.

The spectrum plot in Fig. 4 shows a barely convex shape with variability. The turnover of the upper envelope lies somewhere between $10 \mathrm{GHz}$ and $90 \mathrm{GHz}$ and the source is very bright up to $352 \mathrm{GHz}$.

B1349-439: this is a poorly studied southern source. Its redshift is unknown among its other properties. The GPS shape of the spectrum was noticed by Tornikoski et al. (2001).

In our plot in Fig. 5 there are data at $1-10 \mathrm{GHz}$ and the slope of the spectrum is positive. The high-frequency end of the spectrum shows signs of variability and the spectrum is probably inverted only during flares. The lack of data at $10-90 \mathrm{GHz}$ prevents us from determining the true shape of the lower envelope.

B1546+027: this equatorial source lies at a redshift of 0.413 (Donato et al. 2001). It is a HPQ with maximum optical polarization of $13 \%$ (Smith et al. 1994).

Our data are quite numerous (Fig. 5) and there is variability at all the radio frequencies. The spectrum is inverted during flares, otherwise it is remarkably flat all the way up to $230 \mathrm{GHz}$. The variability is not great enough for an idb classification.

B1637+574: the optical polarization of this quasar is $1.20 \%$ and the redshift 0.750 (Wills \& Wills 1976). The average radio polarization is $2.28 \%$ at $14.5 \mathrm{GHz}$ according to Aller et al. (2003).
This source has not exactly been classified as a GPS source but Rudnick \& Jones (1982) reported that it has a convex radio spectrum and also stated that, based on their study, sources with simple-convex spectra are "relatively quiescent". We strongly disagree with the classification: our plot (in Fig. 5) does not show a convex-spectrum, relatively quiescent source but a source with flat spectrum and conspicuous variability $\left(\operatorname{Var}_{\Delta S}(37 \mathrm{GHz})=5.62\right)$.

B1749+096: this object is also known as OT +081 and $4 \mathrm{C}+09.57$. It is a BL Lac object with an optical polarization of $32 \%$ (Fan \& Lin 1999) and a redshift of 0.322. Gabuzda et al. (1999) made VLBI observations of B1749+096 obtaining a map of a double structure with a smoothly curved jet having weak components. Liu \& Zhang (2002) report the jet to be only $10 \mathrm{pc}$ in projected length.

The continuum spectrum in Fig. 5 is clearly inverted in the maxima, with the turnover at $22-100 \mathrm{GHz}$. The minima are very flat up to $100 \mathrm{GHz}$. The variability at the high frequencies is outstanding: the quiescent flux density is less than $1 \mathrm{Jy}$ and during the outbursts the values reach up to over $10 \mathrm{Jy}$ $\left(\operatorname{Var}_{\Delta S}(37 \mathrm{GHz})=13.21\right.$ and $\left.\operatorname{Var}_{\Delta S}(90 \mathrm{GHz})=18.16\right)$. Dallacasa et al. (2000) classified this source as an HFP but we suggest that it is only a flat-spectrum source with inverted spectrum during flares.

B1828+487: this is a CSS quasar with a turnover frequency of $<20 \mathrm{MHz}$ (e.g. Spencer et al. 1989) at $z=0.692$ that has detailed correspondence between the radio and the optical images of the nucleus and the hotspots (de Vries et al. 1999). It is one of the brightest sources in the $3 \mathrm{C}$ catalog, where it is called 3C 380. It has been discussed (e.g. Wilkinson et al. 1991) whether this source is intrinsically small (jets of $2.6 \mathrm{kpc}$ Liu \& Zhang 2002) or completely surrounded by a halo of $60 \mathrm{kpc}$ in diameter (Wilkinson et al. 1991) and a representative of the CSS class or is it a Fanaroff-Riley type II galaxy viewed from end-on. The superluminal motion found by Wilkinson et al. (1991) support the latter but the comparatively weak and nonvariable core is inconsistent with it. The optical polarization of this source is low: only $0.89 \%$ (Wills et al. 1992).

Again our data (Fig. 5) lacks the low frequency end of the spectrum so the possible turnover at low MHz-frequencies cannot be verified. There are only minor variations at high frequencies, in good agreement with the properties observed by Wilkinson et al. (1991).

B1936-155: this blazar has a redshift of 1.657 (Jauncey et al. 1984). It is classified as a high-peaking GPS source (Tornikoski et al. 2001). The spectrum in Fig. 5 has a slightly convex shape at the maxima but there is variability at all radio frequencies and the lower envelope is flat.

B1954-388: this source is an optically violently variable quasar with a redshift of 0.626 and an optical polarization of $11 \%$ (Shen et al. 1998). Figure 5 presents the spectrum of this source. With the current dataset it is more flat than gigahertzpeaked as it was classified earlier by us (Tornikoski et al. 2001). The lower envelope is very flat up to the highest observed frequency.

B2008-159: the redshift of this low polarization quasar is 1.181. This source was identified as a extreme-GPS source in 

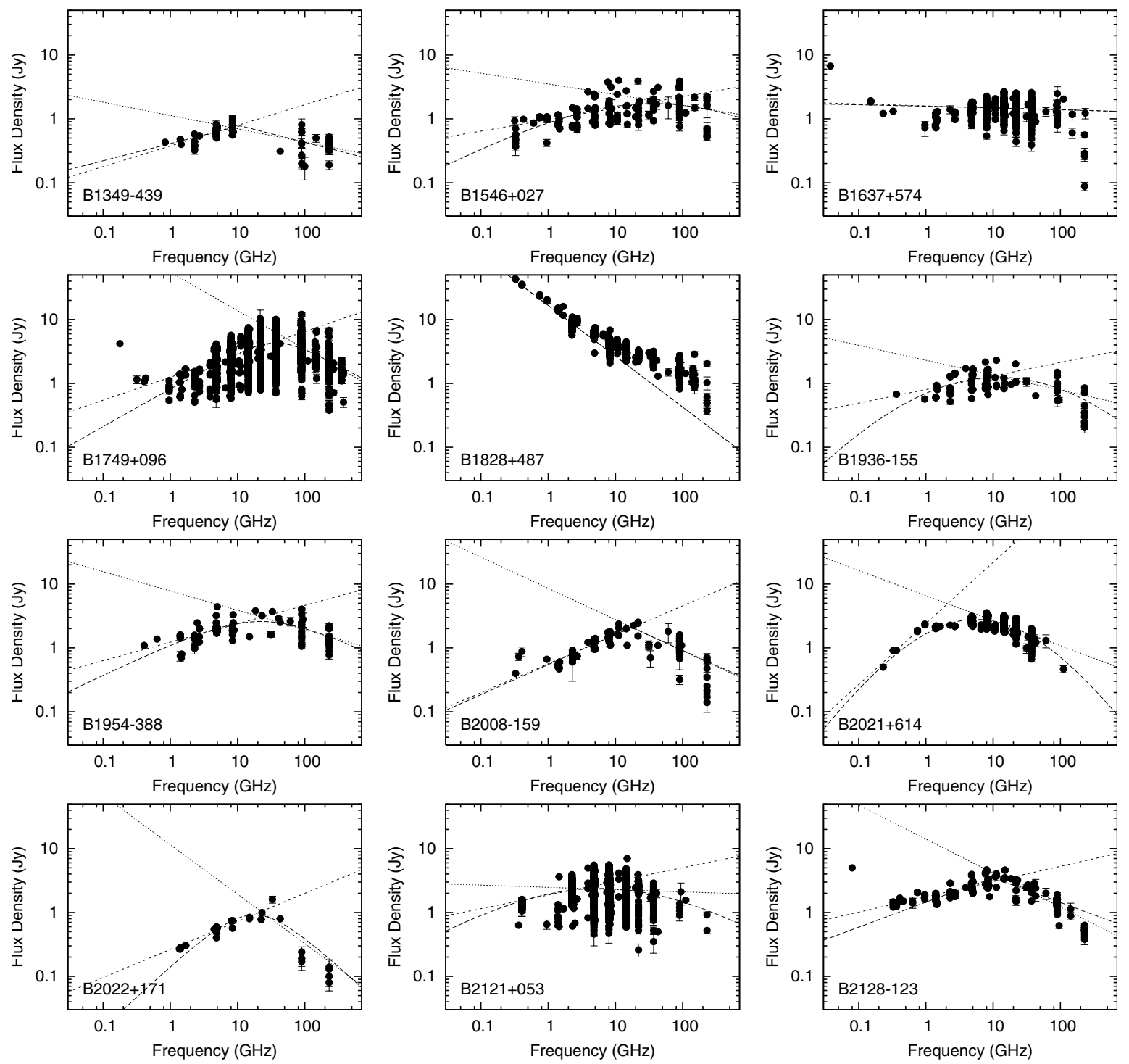

Fig. 5. Radio spectra of the previously identified inverted-spectrum sources.

Tornikoski et al. (2000). Our plot in Fig. 5 shows a convex, variable spectrum.

B2021+614: this radio galaxy, aka OW 637, is at $z=$ 0.2266 and has a core, $40 \mathrm{pc}$ in diameter (Bartel et al. 1984). It has been listed as a GPS source by O'Dea et al. (1991). This object is a peculiar case; its optical properties are typical for radio galaxies but its radio behaviour and spectral line properties are more like those of quasars and BL Lacs (Bartel et al. 1984).

The spectrum in Fig. 5 is inverted below $1 \mathrm{GHz}$ and very flat between $1 \mathrm{GHz}$ and $10 \mathrm{GHz}$. There is very little variability at e.g. $4.8 \mathrm{GHz}$ from the UMRAO: the fractional variability index is 0.34 over a monitoring period of 20 years. The spectrum steepens right after $10 \mathrm{GHz}$ and there is some variability at the high frequencies $\left(\operatorname{Var}_{\Delta S}(37 \mathrm{GHz})=2.00\right)$. Aller et al. 2003 found also that "the source exhibits well defined variability while maintaining its GPS spectral shape." This can be considered as a genuine GPS source.

B2022+171: this is a relatively little studied low polarization quasar. It has a redshift of 1.05 (Veron 1996). The source has been identified as a HFP by Dallacasa et al. (2000).
The datapoints in Fig. 5 are aligned into a convex shape but more data is needed. The variability index at $37 \mathrm{GHz}$ is 2.33 so it is possible that the convex shape of the spectrum is only due to an outburst occurring when the low frequency observations were made.

B2121+053: the quasar has a redshift of $z=1.878$ (Hewitt $\&$ Burbidge 1989). It has been identified as a HFP by Dallacasa et al. (2000). In our plot the lower envelope of the spectrum is flat and the upper envelope is clearly convex. The variability is extremely high: $\operatorname{Var}_{\Delta S}(22 \mathrm{GHz})=11.35$.

B2128-123: this source was identified as a GPS source by Tornikoski et al. (2001). The present spectrum in Fig. 5 is slightly convex and there is some variability.

B2134+004: this source is a quasar with a redshift of 1.932 (Osmer et al. 1994) and low polarization $\left(p_{\text {opt }}=0.5\right.$, Wills et al. 1992). The VLBA observations have shown that there are two unresolved components separated only by 1.7 mas, corresponding 14 pc (Taylor 2000).

The spectrum (in Fig. 6) is elegantly inverted even though there is some variability at all frequencies. Kovalev et al. (2000) 

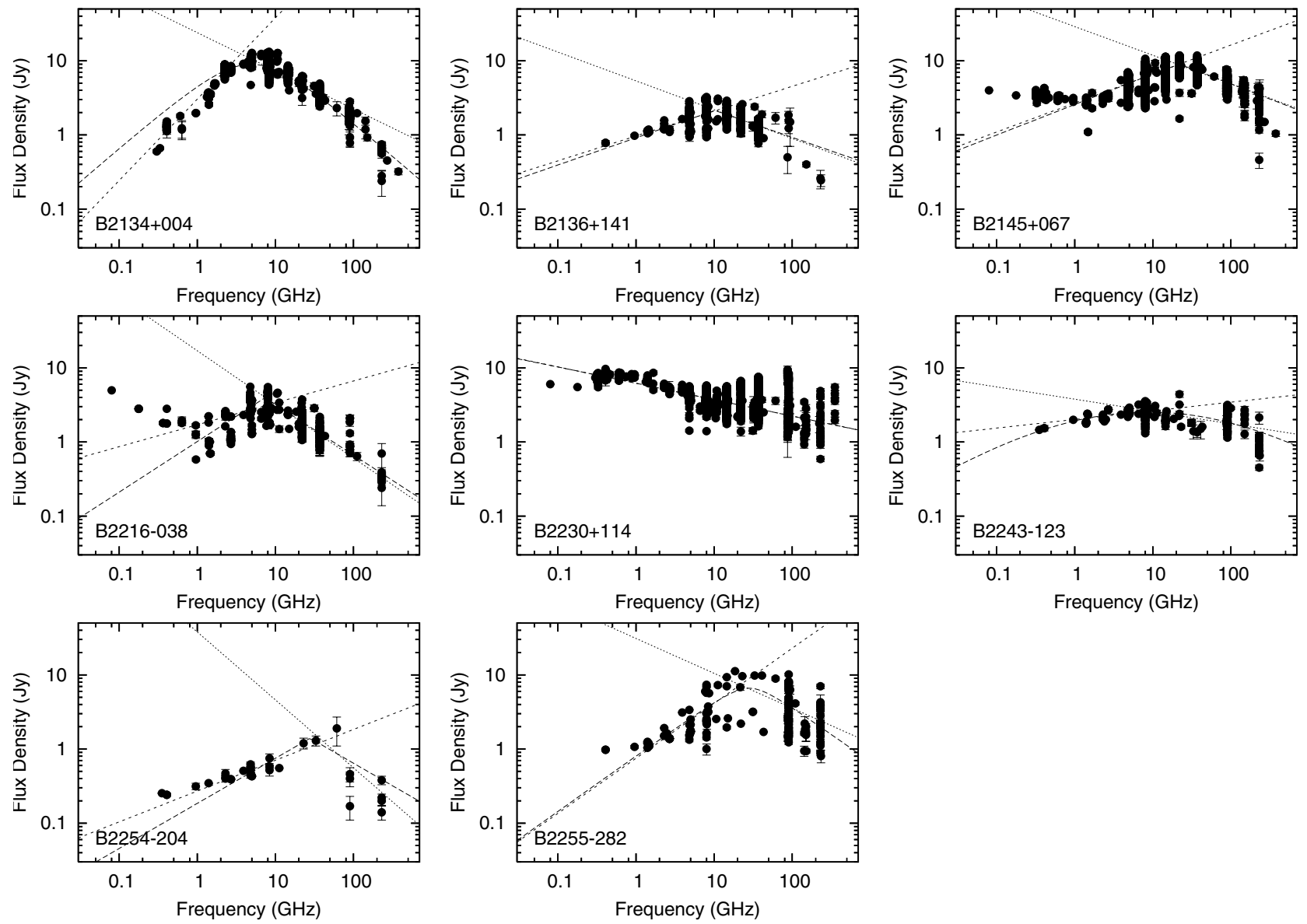

Fig. 6. Radio spectra of the previously identified inverted-spectrum sources.

observed this source with RATAN-600 telescope simultaneously at six radio frequencies obtaining very convex, just little varying spectra for eight epochs during three years. In our plot, the spectral indices are +1.1 and -0.51 below and above the turnover, respectively. O'Dea, (1998) classified this source as a GPS sources and Dallacasa et al. (2000) as a HFP. There is variability but it stays below our limit of $\operatorname{Var}_{\Delta S \text {, max }}<3$ and thus the source is one of the few sources with genuine GPS properties in this sample.

B2136+141: this LPQ $(z=2.427)$ was identified as a GPS source by Tornikoski et al. (2001). It has a spectrum which is flat at quiescent levels and slightly convex during bursts.

B2145+067: earlier we found this source to have an inverted spectrum (Tornikoski et al. 2001) but the current spectrum plot with our newest data points presents a variable spectrum which is convex only during outbursts.

B2216-038: the quasar has a redshift $z=0.901$ and a polarization of $1.26 \%$ (Wills et al. 1992). Its spectrum (in Fig. 6) is convex with a turnover frequency of $7.4 \mathrm{GHz}$. There seems to be an equal proportion of variability in the optically thin and thick parts. The variability indices are $\operatorname{Var}_{\Delta S}(\sim 5 \mathrm{GHz})=3.35$ and $\operatorname{Var}_{\Delta S}(37 \mathrm{GHz})=2.53$.

B2230+114: aka CTA 120, is strong at all wavelengths from radio to $\gamma$-rays (Lin et al. 1996). It has an optical polarization degree of $7.32 \%$ (Wills et al. 1992). It lies at a redshift of 1.037 and there is superluminal motion in a wiggling one-sided jet (Taylor et al. 2000). Liu \& Zhang (2002) report the jet to be $110 \mathrm{pc}$ in projected length. The source has been mentioned to be a CSS source with a turnover frequency of $0.9 \mathrm{GHz}$ (Spencer et al. 1989) and a GPS source (Dallacasa et al. 1995). Rantakyrö et al. (2003) presented results of interferometry and long term monitoring studies and concluded that the flux density, the spectral index and the turnover frequency vary rapidly corresponding the structural changes observed with interferometry.

The spectrum is very flat up to $100 \mathrm{GHz}$ and there is considerable variability at all the monitoring frequencies.

B2243-123: this HPQ has a redshift of 0.63 and the radio spectrum was found to be convex by Tornikoski et al. (2001). Data form the UMRAO show that the lower envelope is flat; the upper envelope is very slightly convex but there is less variability than in most of the sources with inverted spectra during bursts so this is labelled as a flat-spectrum source.

B2254-204: this BL Lac object has a convex spectrum and variability at least at high frequencies. Probably there is variability also at the low frequencies and the spectrum is truly flat and inverted only during bursts.

B2255-282: is a LPQ and has been identified as a highpeaking GPS source by Tornikoski et al. (2001). The lower envelope of the spectrum in flat but the upper envelope is clearly convex with a turnover at $22-37 \mathrm{GHz}$. Variability is conspicuous: $\operatorname{Var}_{\Delta S}(90 \mathrm{GHz})=7.21$. 

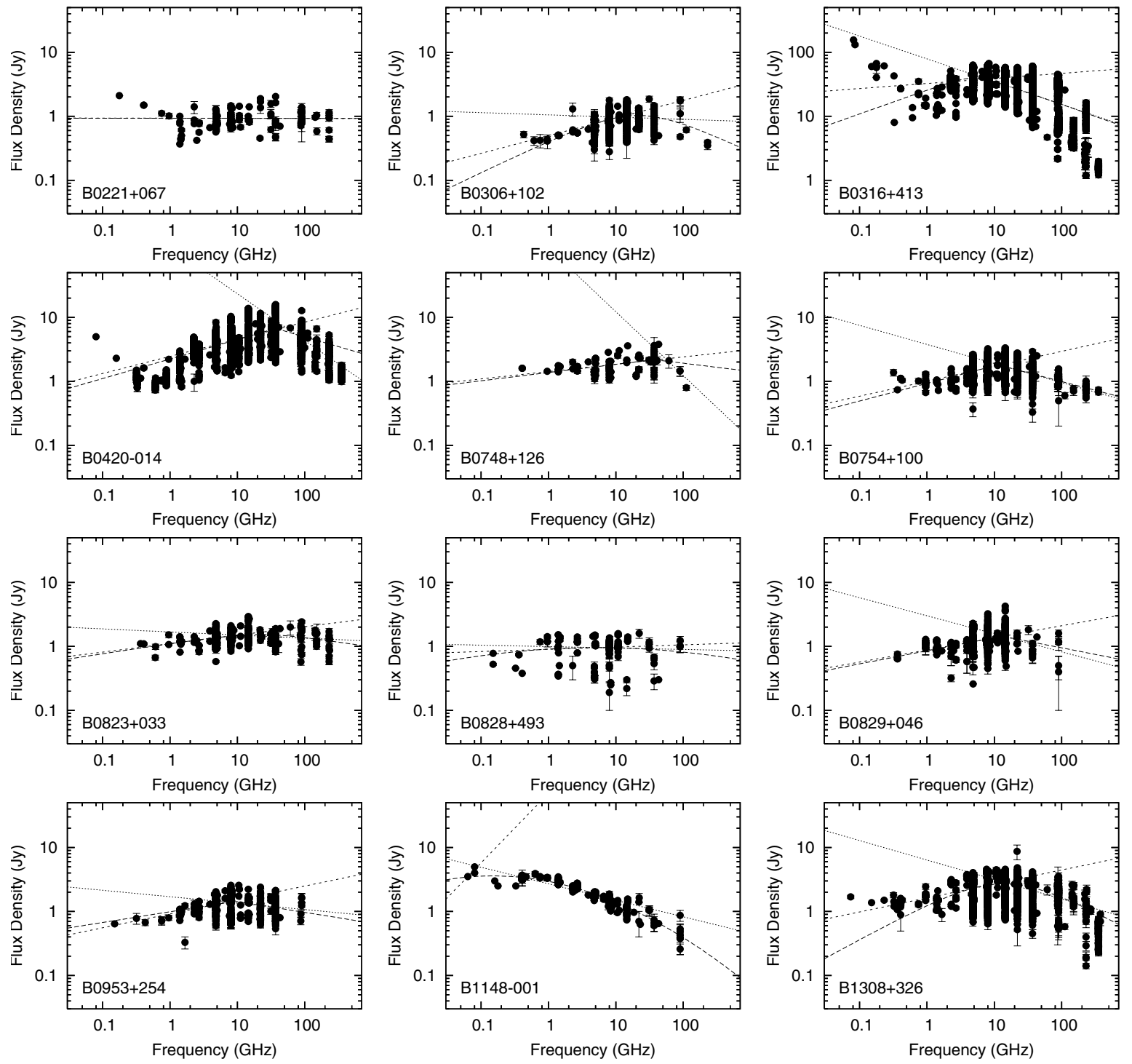

Fig. 7. Radio spectra of the candidate inverted-spectrum sources.

\subsection{Candidate inverted-spectrum sources}

B0221+067: is a highly polarized quasar at $z=0.511$. The overall shape of the spectrum in Fig. 7 is flat and the fit was not able to find any turnover in the data.

B0306+102: this source is a quasar having a redshift of $z=$ 0.863. Zirbel \& Baum (1998) noted that this source is originally classified as a BL Lac, but that Veron (1994) suggested that it would be a quasar due to its spectroscopic characteristics. In our plot in Fig. 7 the upper envelope is quite flat up to $90 \mathrm{GHz}$.

B0316+413: better known as Perseus A, 3C 84 and NGC 1275, this source is one of the most studied radio galaxies due to its peculiar properties. It was studied by Carl Seyfert (1943) who classified it as one of the first Seyfert II-galaxies but later it has also been classified as a BL Lac object (Marcha et al. 1996). It is in the center of the Perseus cluster at a redshift of 0.0172 and it is considered to be a colliding system (de Koff et al. 2000). The source exhibits strong far-infrared emission as well as extended radio and X-ray emission (Goudfrooij et al. 1994). Liu \& Zhang (2002) report of two jets associated with this source, each $5 \mathrm{kpc}$ in projected length, so the projected overall size of $3 \mathrm{C} 84$ is of the order of $10 \mathrm{kpc}$. Optical polarization of this source is $1.6 \%$ (Nartallo et al. 1998).

The overall flux density of B0316+413 has been decreasing slowly and monotonically during the decades (Peng et al. 2000). This is confirmed by the frequent monitoring data from Metsähovi: 3C 84 is used as one of the secondary calibrators and pointing sources in the monitoring campaigns as well as in our observations.

In the spectrum seen in Fig. 7 the some convexity can be seen, but the spectral indices from the fit are very flat. There is significant variability at all frequencies.

B0420-014: this source is also known as OA 129 and is a quasar $(z=0.915$ from Peng et al. 2000) with a very high optical polarization of ca $17 \%$ (Wills et al. 1992). It is very variable both in the radio (Peng et al. 2000) and in the optical wavelengths where the steepest detected variation was 0.12 mag in 40 min (Villata et al. 1997). It has also been detected in the $\gamma$ and X-rays (Radecke et al. 1995). 
The high-frequency outbursts of B0420-014 have been studied in detail by Stevens et al. (1995). They used monitoring data from the James Clerk Maxwell Telescope (JCMT), the Metsähovi Radio Observatory, Institut de RadioAstronomie Millimetrique (IRAM) and the SEST to study the evolution of the bursts at several frequencies between $22 \mathrm{GHz}$ and $375 \mathrm{GHz}$. The behaviour of the flares was observed to be qualitatively consistent with the shocked jet model by Marscher \& Gear (1985).

The spectral shape is convex regardless of the intense variability on both sides of the turn over $\left(\operatorname{Var}_{\Delta S}(\sim 5 \mathrm{GHz})=4.73\right.$ and $\left.\operatorname{Var}_{\Delta S}(90 \mathrm{GHz})=8.12\right)$

B0748+126: this object is a low polarization quasar $\left(p_{\text {opt }}=\right.$ 1.4\% Wills et al. 1992) with two suggested redshifts: $z=0.281$ or $z=0.880$ (Wills \& Wills 1976). A redshift of 0.889 is favoured by Liu \& Zhang (2002). According to them this source has a single jet of $61 \mathrm{kpc}$. The core remains unresolved in the global $3 \mathrm{~mm}$ VLBI observations and a faint component found in the observations is 0.37 mas away from the core (Rantakyrö et al. 1998).

The shape of the spectrum in Fig. 7 is flat but the maximum values are slightly inverted up to $37 \mathrm{GHz}$ and decline towards the higher frequencies. Based on these data the source is a flat-spectrum source with an inverted spectrum during the outbursts.

B0754+100: Tapia et al. (1977) identified this source as a BL Lac object. This is one of the sources which looked promisingly inverted in an early phase of our study but as data were collected the shape of the spectrum turned out to be flat in the quiescent levels and inverted during bursts.

B0823+033: is a BL Lac object $(z=0.506)$. It is a variable source with a beautifully flat spectrum all through the observing frequencies.

B0828+493: this is a BL Lac object at a redshift of 0.548 . The upper envelope of its spectrum is perfectly flat and there is variability at all the radio frequencies.

B0829+046: is also a BL Lac object $(z=0.18)$. The spectrum is flat except the data from the UMRAO extending over 26 years which show convex shape in the maximum spectrum.

B0953+254: the redshift of this object is 0.712 (Liu \& Zhang 2002). Its optical polarization is $1.29 \%$ (Wills et al. 1992). The spectrum of the source (in Fig. 7) is slightly inverted during bursts and very flat at quiescent times.

B1148-001: the spectrum of this distant $(z=1.983)$ quasar is flat.

B1308+326: the nature of this source is somewhat unclear. It has been classified as a BL Lac object (e.g. Stickel et al. 1993) but Gabuzda et al. (1993) suggested it to be a quasar with very weak emission lines. The optical polarization of the source is $10.2 \%$ (Wills \& Wills 1976). Its redshift is 0.996 (Wills \& Wills 1976). Stickel et al. (1993) report a faint nearby companion galaxy 5 arcseconds from the source. Our plot shows that this source is a flat-spectrum source with inverted spectrum during bursts and during the quiescent state the spectrum becomes steeper.

B1642+690: this source is a core dominated quasar at $z=0.751$ (Venturi et al. 1997). Pearson et al. (1986) found the source to have superluminal motion even up to $9.3 \mathrm{c}$.
Venturi et al. (1997) monitored the source with VLBI at $6 \mathrm{~cm}$ obtaining maps of parsec-scale structures and confirming the superluminal motion. They found an inverted-spectrum core and a single, bent jet aligned only ca $12^{\circ}$ from the line of sight. Their contour plots showed several components or knots in the jet moving away from the core.

The median spectrum is very flat up to ca $100 \mathrm{GHz}$ and the variability produces a clearly convex shape of the upper envelope.

B1730-130: this variable quasar lies at redshift 0.902. Its quiescent spectrum is declining but the upper envelope is slightly convex with a peak at $10-22 \mathrm{GHz}$.

B1741-038: this quasar has a redshift of 1.057 (Veron-Cetty \& Veron 1993). Fey et al. (1996) observed the source with VLBI and found it to be a simple compact source with two components. Also some weak extended emission was observed.

This is similar case to e.g. B1546+027: the minima are flat up to $150 \mathrm{GHz}$, they seem to be mildly inverted and the maxima have a clear convex shape.

B2131-021: the redshift of this BL Lac object is 1.285. Cassaro et al. (1999) made VLBI observations with the VLA of a sample of 28 BL Lacs including this object. The source is compact but there is some extended emission up to angular size of $9^{\prime \prime}$.

Lower envelope of the spectrum in Fig. 8 is flat. There is variability at all the frequencies and the upper envelope is barely convex.

\section{Discussion}

When initially selecting sources for this sample we found many promising GPS candidates and we believed that we were going to identify several new high-peaking GPS sources. But as the study progressed and we gained more data, the true nature of the variability of these sources became evident and most of our candidates turned out to be just flat-spectrum sources with high-frequency flares. Interestingly, with our long term monitoring data the same thing happened with most of the GPS sources taken from the literature.

Only five sources (B0741-063, B0742+103, B0743-006, B2021+614, B2134+004) still seem to comply with the original GPS classification of very low variability and a convex quiescent spectrum. Two more sources, B0552+398 and B2008-159 have nicely convex spectra but have too flat spectral indices or show prominent variability in the high-frequency tail of the spectrum, contradicting to the original GPS classification. All the other GPS sources from the literature turned out to be either significantly variable, or relatively ordinary flatspectrum sources with inverted spectra only during flares. In the latter case the initial GPS classification had probably been based on too sparse data, whereas long-term monitoring reveals the actual shape of the spectrum during the true quiescent as well as flaring stages.

Our result is different from the result of variability studies of Jauncey et al. (2003) who found only 10\% of GPS sources to vary. In our sample the previously identified GPS sources exhibited remarkable variability: 14 sources $(53.8 \%)$ have a 

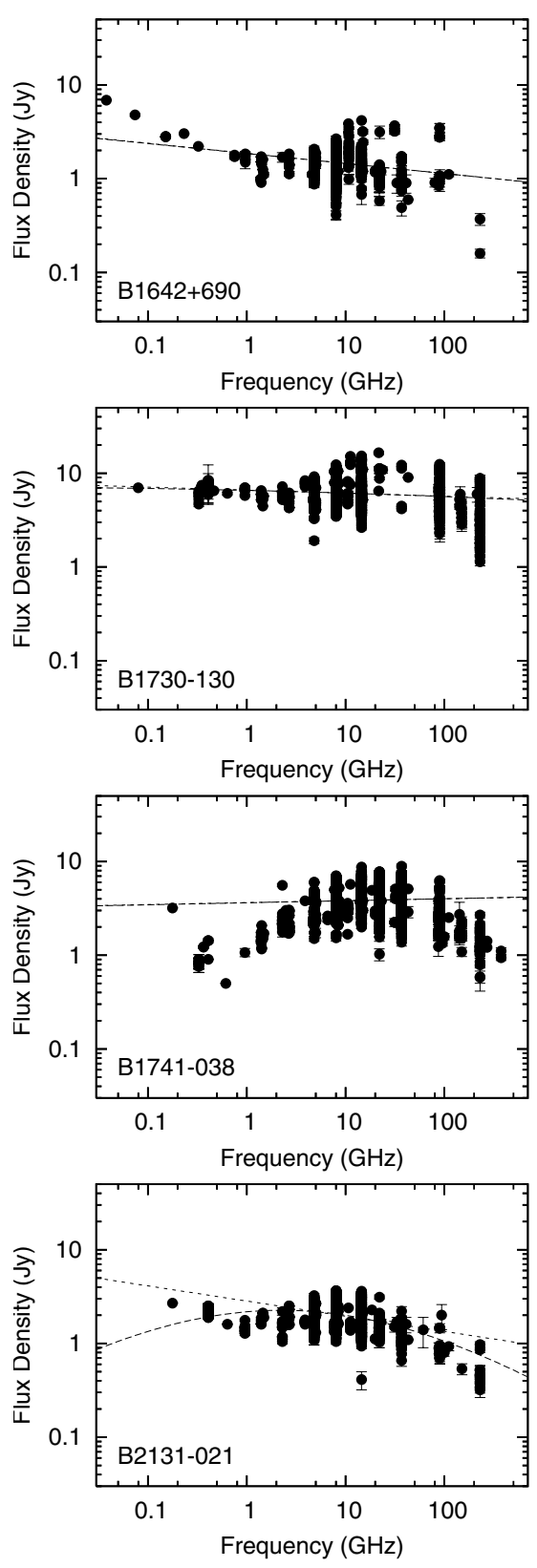

Fig. 8. Radio spectra of the candidate inverted-spectrum sources.

fractional variability index greater than 3 and nine sources $(34.6 \%)$ have $1.5<\operatorname{Var}_{\Delta S}<3$, which Jauncey et al. would probably consider to be strong variability. None of the previously identified GPS sources with more than one data point at each frequency have a maximum variability index smaller than 1 i.e. the variations are greater than $100 \%$ of the smallest flux density value. One of our primary criteria was low variability when selecting the genuine GPS sources among the sample. Compared to the variability of the rest of the sources in our sample, $\operatorname{Var}_{\Delta S}=3$ can be considered to be relatively low even though it means variability of $300 \%$ of the lowest flux density value. This can also be visually verified from the spectrum plots.

Two examples of different types of sources are presented in Fig. 9. B0743-006 is a classical GPS source with low variability and always convex shape of the spectrum with almost constant turnover frequency. When having a burst B0528+134 has a simple convex spectrum and can be mistaken as a GPS source but the shape of the spectrum evolves with the activity and at least the low frequency end of the spectrum can be very flat at the quiescent state. The change in the shape of the spectrum at different levels of activity is very interesting and worth a deeper look which is beyond the scope of this paper and which we intend to take in a forthcoming paper.

Based on our sample we conclude that genuine quasar-type GPS sources are relatively rare, whereas sources that are variable and can have inverted spectra especially during outbursts are much more common. Because many of the "bona fide" GPS sources from the literature are now observed to be variable flatspectrum sources, the whole concept of GPS sources should be re-examined.

If the GPS class is to consist of only sources complying with the classical GPS properties, a large number of sources identified as GPS sources in the literature are misclassified. We suggest that the term GPS should be reserved for the small number of sources that systematically maintain a convex continuum spectrum and show relatively low variability. In these sources neither the turnover frequency nor the general shape of the spectrum changes with time.

The sources that we have now classified as being "sources that are inverted during flares" are sources in which strong variability is observed especially at the higher frequencies, causing the continuum spectrum to become inverted. In some of the sources the turnover frequency during their activity states can be as high as ca. $100 \mathrm{GHz}$. During the more quiescent states their spectra, however, are flat or even falling. At least the shape of the high-frequency part of these spectra can be explained by radio shocks that cause the variability and which are first observed at the higher frequencies (Valtaoja et al. 1992; Tornikoski et al. 2005, in preparation for A\&A). The turnover frequency decreases with time, as the shocked material expands and the radio shock finally decays. In some cases there may be several superposed shock components observed at the same time, especially at the lower frequencies where the events are usually slower and delayed in comparison to the millimetredomain, making the shape of the continuum spectrum and its evolution in time more complicated.

Our result is in accordance with the suggestion of Snellen et al. (1999) that GPS quasars are related to flat-spectrum radio sources rather than GPS galaxies. We suggest that at least many of the earlier GPS identifications are in reality flat-spectrum sources that were observed during a high-frequency flare when the spectrum was temporarily inverted. Thus long-term multifrequency monitoring of these sources is essential in order to distinguish between the true GPS sources and the highly variable flat-spectrum sources that sometimes have high to extreme-peaking continuum spectra. We will discuss the implications of these present results and the theoretical interpretation of the various types of sources in a sequel to this observational paper (Torniainen et al. 2005, in preparation).

One of our objectives for this study was to better understand the extragalactic foreground sources that could be bright in the Planck satellite's frequency range and we were especially interested in the high-frequency peaking sources. Thus the 

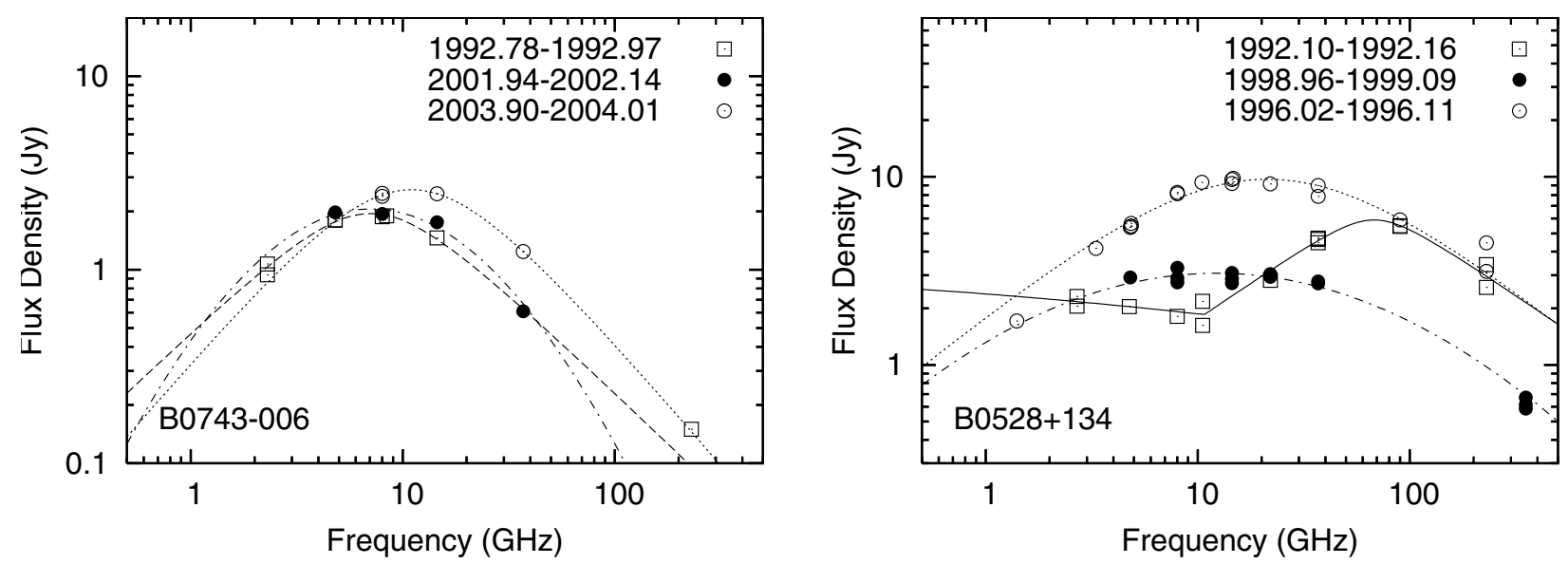

Fig. 9. Semisimultaneous spectra of two different types of sources: B0743-006, which has low variability and the shape of the spectrum does not depend on the state of activity; and B0528+134, which has a clearly inverted spectral shape in the active phase but rather flat in more quiescent phases. For B0528+134, epoch 1992.10-1992.16, two separate fits have been combined in order to better model the shape of the spectrum (solid line).

outcome of this study should also be considered when estimating the number of the bright sources in the Planck foreground. Whereas the number of true, consistently inverted, highpeaking spectra seems to be relatively low, the number of flatspectrum sources that can be very bright even in the millimetredomain during their high activity states can be much larger than usually expected. The fact that they are very variable makes it more complicated to estimate their fluxes at any given time, emphasizing the importance of radio variability models, statistical studies for "educated guesses", as well as dense monitoring of such candidate sources for the actual knowledge of their flux levels. Our group is in the process of analyzing highfrequency radio data of a large sample of sources in order to better understand the millimetre-to-centimetre variability behaviour of AGNs (Tornikoski et al. 2005, in preparation). In order to better understand the millimetre-domain variability of various AGN types we have also extended our radio monitoring campaigns to include sources that have earlier rarely, or never before, been observed at the high radio frequencies.

\section{Conclusions}

Our primary goal in this study was to identify new highpeaking GPS sources and to study the variability of the known quasar-type GPS sources. We combined data from our observing campaigns and the literature to construct the radio continuum spectra for the sample of 35 "bona fide" inverted-spectrum sources and 16 candidate GPS sources and 9 sources with fairly convex spectrum.

The long term monitoring data from the UMRAO and the Metsähovi Radio Observatory revealed significant amount of variability in most of the "bona fide" GPS sources which is in contradiction with the original GPS definition. Also the shape of the spectra remained clearly convex for only a fraction of these sources. Only five of the 26 GPS sources still remained at an almost constant flux level at all times with inverted spectrum and can yet be classified as GPS sources. Most of the sources in the sample turned out to be inverted but very variable or variable flat-spectrum sources with inverted spectral shape only during flares (12 sources). There were also sources with spectra that were simply flat or steeply falling.

In many cases the classification for the "bona fide" GPS sources has earlier too easily been done with too sparse data and there are plenty of source identified as GPS sources which truly are variable flat-spectrum sources. The characteristics of the GPS sources have been interpreted from samples of sources misidentified as GPS sources and thus the whole concept of GPS quasars should be reassessed. According to our study the genuine quasar-type GPS sources seem to be very rare.

Acknowledgements. Our group's observing projects at Metsähovi and SEST were funded by the Academy of Finland, which we gratefully acknowledge. The UMRAO is partially supported by funds from the NSF and from the University of Michigan Department of Astronomy. The SEST was operated jointly by ESO and the Swedish National Facility for Radio Astronomy, Onsala Space Observatory at Chalmers University of Technology. The authors made use of the SIMBAD database, operated at CDS, Strasbourg, France and the database CATS (Verkhodanov et al. 1997) of the Special Astrophysical Observatory. This research has also made use of the NASA/IPAC Extragalactic Database (NED) which is operated by the Jet Propulsion Laboratory, California Institute of Technology, under contract with the National Aeronautics and Space Administration and NASA's Astrophysics Data System. I.T. acknowledges a grant from Vilho, Yrjö ja Kalle Väisälä Foundation for part of this work.

\section{References}

Aller, H. D., Aller, M. F., Latimer, G. E., \& Hodge, P. E. 1985, ApJS, 59,513

Aller, M. F., Aller, H. D., \& Hughes, P. A. 1992, ApJ, 399, 16

Aller, M. F., Aller, H. D., \& Hughes, P. A. 2003, ApJ, 585, 33

Baars, J. W. M., Genzel, R., Pauliny-Toth, I. I. K., \& Witzel, A. 1977, A\&A, 61, 99.

Bartel, N., Shapiro, I. I., Huchra, J. P., \& Kühr, H. 1984, ApJ, 279, 112

Barthel, P. D., Pearson, T. J., \& Readhead, A. C. S. 1988, ApJ, 329, 51

Baum, S. A., O’Dea, C. P., de Bruyn, A. G., \& Murphy, D. W. 1990, A\&A, 232, 19 
Beasley, A. J., Conway, J. E., Booth, R. S., Nyman, L.-A., \& Holdaway, M. 1997, A\&AS, 124, 469

Becker, R. H., White, R. L., \& Edwards, A. L. 1991, ApJS, 75, 1

Bondi, M., Padrielli, L., Fanti, R., et al. 1996, A\&AS, 120, 89

Browne, I. W. A., Patnaik, A. R., Walsh, D., \& Wilkinson, P. N. 1993, MNRAS, 263, 32

Cassaro, P., Stanghellini, C., Bondi, M., et al. 1999, A\&AS, 139, 601

Condon, J. J., Yin, Q. F., Thuan, T. X., \& Boller, Th. 1998, AJ, 116, 2682

Dagkesamanskii, R. D., Samodurov, V. A., \& Lapaev, K. A. 2000, ARep, 44, 18

Dallacasa, D., Fanti, C., Fanti, R., Schilizzi, R. T., \& Spencer, R. E. 1995, A\&A, 295, 27

Dallacasa, D., Stanghellini, C., Centonza, M., \& Fanti, R. 2000, A\&A, 363,887

Djorgovski, S., Perley, R., Meylan, G., \& McCarthy, P. 1987, ApJ, 321,17

de Koff, S., Best, P., Baum, S. A., et al. 2000, ApJS, 129, 33

de Vries, W. H., Barthel, P. D., \& O’Dea, C. P. 1997, A\&A, 321, 105

de Vries, W. H., O’Dea, C. P., Baum, S. A., \& Barthel, P. D. 1999, ApJ, 526, 27

Donato, D., Ghisellini, G., Tagliaferri, G., \& Fossati, G. 2001, A\&A, 375,739

Douglas, J. N., Bash, F. N., Bozyan, F. A., Torrence, G. W., \& Wolfe, C. 1996, AJ, 111, 1945

Drinkwater, M. J., Webster, R. L., Francis, P. J., et al. 1997, MNRAS, 284,85

Edelson, R. A. 1987, AJ, 94, 1150

Edge, A. C., Jones, M., Saunders, R., Pooley, G., \& Grainge, K. 1996, Proc. Second Workshop on Gigahertz Peaked Spectrum Sources and Compact Steep Spectrum Sources, ed. I. A. G. Snellen, R. T. Schilizzi, H. J. A. Röttgering, \& M. N. Bremer (Leiden: Leiden Observatory), 43

Edge, A. C, Pooley, G., Jones, M., Grainge, K., \& Saunders, R. 1998, IAU Colloq., 164, Radio emission from Galactic and Extragalactic Compact Sources, ed. J. A. Zensus, G. B. Taylor, \& J. M. Wrobel (San Francisco: ASP), ASP Conf. Ser., 144, 187

Falomo, R., Scarpa, R., \& Bersanelli, M. 1994, ApJS, 93, 125

Fan, J. H., \& Lin, R. G. 1999, ApJS, 121, 131

Fanti, C., Fanti, R., Parma, P., Schilizzi, R. T., \& van Breugel, W. J. M. 1985, A\&A, 143

Fanti, R., Fanti, C., Schilizzi, R. T., et al. 1990, A\&A, 231, 333

Fanti, C., Fanti, R., Dallacasa, D., et al. 1995, A\&A, 302, 317

Fey, A. L., Clegg, A. W., \& Fiedler, R. L. 1996, ApJ, 468, 543

Gabuzda, D. C., Kollgaard, R. I., Roberts, D. H., \& Wardle, J. F. C. 1993, ApJ, 410, 39

Gabuzda, D. C., Pushkarev, A. B., \& Cawthorne, T. V. 1999, MNRAS, 307,725

Gabuzda, D. C., Kochenov, P. YU., \& Cawthorne, T. V. 2000, MNRAS, 319, 1125

Gear, W. K., Stevens, J. A., Hughes, D. H., et al. 1994, 267, 167

Geldzahler, B. J., \& Witzel, A. 1981, AJ, 86, 1306

Ghosh, T., Gopal-Krishna, \& Rao, A. P. 1994, A\&AS, 106, 29

Gopal-Krishna, \& Wiita, P. J. 1991, ApJ, 373, 325

Goudfrooij, P., Hansen, L., Jorgensen, H. E., \& Norgaard-Nielsen, H. U. 1994, A\&AS, 105,341

Grainge, K., \& Edge, A. 1998, in Proc. 33d Recontres de moriong, Fundamental Parameters in Cosmology, ed. J. Tran Thanh Van, Y. Giraud-Heraud, \& F. Bouchet (Gif-sur-Yvette: Éditions Frontières), 151

Gregory, P. C., \& Condon, J. J. 1991, ApJS, 75, 1011

Gregory, P. C., Vavasour, J. D., Scott, W. K., \& Condon, J. J. 1994, ApJS, 90, 173
Griffith, M. R., Wright, A. E., Burke, B. F., \& Ekers, R. D. 1995, ApJS, 97, 347

Hewitt, A., \& Burbidge, G. 1989, ApJS, 69, 1

Hewitt, A., \& Burbidge, G. 1993, ApJS, 87, 451

Hummel, C. A., Schalinski, C. J., Krichbaum, T. P, Witzel, A., \& Johnston, K. J. 1988, A\&A, 204, 68

Hunter, S. D., Bertsch, D. L., Dingus, B. L., et al. 1993, ApJ, 409, 134

Hutchings, J. B. 1992, AJ, 104, 1311

Jauncey, D. L., Batty, M. J., Wright, A. E., Peterson, B. A., \& Savage, A. 1984, ApJ, 286, 498

Jauncey, D. L., King, E. A., Bignall, H. E., et al. 2003, PASA, 20, 151

Jeyakumar, S., Saikia, D. J., Pramesh Rao, A., \& Balasubramanian, V. 2000, A\&A, 362, 27

Kellermann K. I., Vermeulen, R. C., Zensus, J. A., \& Cohen, M. H. 1998, AJ, 115, 1295

Kovalev, Y. Y., Nizhelsky, N. A., Kovalev, Yu. A., et al. 1999, A\&AS, 139,545

Kovalev, Yu. A., Kovalev, Y. Y., \& Nizhelsky, N. A. 2000, PASJ, 52, 1027

Kühr, H., Witzel, A., Pauliny-Toth, I. I. K., \& Nauber U. 1981, A\&AS, 45,367

Large, M. I., Cram, L. E., \& Burgess, A. M. 1991, Obs., 111, 72

Lawrence, C. R., Bennett, C. L., Garcia-Barreto, J. A., Greenfield, P. E., \& Burke, B. F. 1983, ApJS, 51, 67

Lister, M. L., \& Marscher, A. P. 1996, BAAS, 28, 827

Lin, Y. C., Bertsch, D. L., Dingus, B. L., et al. 1996, ApJS, 105, 331

Liu, F. K., \& Zhang, Y. H. 2002, A\&A, 123, 443

Maraschi, A., Schwartz, A., Tanzi, E. G., \& Treves, A. 1085 ApJ, 294, 615

Marcha, M. J. M., Browne, I. W. A., Impey, C. D., \& Smith, P. S. 1996, MNRAS, 281, 425

Marscher, A. P., \& Gear, W. K. 1985, ApJ, 298, 114

Mutel, R. L., \& Phillips, R. B. 1988, International Astronomical Union Symposium, 129, 73

Nartallo, R., Gear, W. K., Murray, A. G., Robson, E. I., \& Hough, J. H. 1998, MNRAS, 297, 667

O’Dea, 1998, PASP, 110, 493

O’Dea, C. P., Stanghellini, C., Baum, S. A., et al. 1990, ApJS, 84, 549

O'Dea, C. P., Baum, S. A., \& Stanghellini, C. 1991, ApJ, 380, 660

Osmer, P. S., Porter, A. C., \& Green, R. F. 1994, ApJ, 436, 678

Owen, F. N., Spanger, S. R., \& Cotton, W. D. 1980, AJ, 85, 351

Pauliny-Toth, I. I. K., Witzel, A., Preuss, E., et al. 1978, AJ, 83, 451

Pearson, T. J., Barthel, P. D., Lawrence, C. R., \& Readhead, A. C. S. 1986, ApJ, 300, 25

Peng, B., Kraus, A., Krichbaum, T. P., \& Witzel, A. 2000, A\&AS, 145, 1

Perley, R. A. 1982, AJ, 87, 859

Radecke, H.-D., Bertsch, D. L., Dingus, B. L., et al. 1995, ApJ, 438, 659

Rantakyro, F. T., Bååth, L. B., Backer, D. C., et al. 1998, A\&AS, 131, 451

Rantakyro, F. T., Wiik, K., Tornikoski, M., Valtaoja, E., \& Bååth, L. B., 2003, A\&A, 405, 473

Reich, W., Reich, P., Pohl, M., Kothes, R., \& Schlickeiser, R. 1998, A\&AS, 131, 11

Reich, W., Fürst, E., Reich, P., et al. 2000, A\&A, 363, 141

Reuter, H.-P., Kramer, C., Sievers, A., et al. 1997, A\&AS, 122, 271R

Robson, E. I., Stevens, J. A., \& Jenness, T. 2001, MNRAS, 327, 751

Rudnick, L., \& Jones, T. W. 1982, ApJ, 255, 39

Salonen, E., Teräsranta, H., Urpo, S., et al. 1987, A\&AS, 70, 409

Schramm, K.-J., Borgeest, U., Kuehl, D., et al. 1994, A\&AS, 106, 349

Seielstad, G. A., Pearson, T. J., \& Readhead, A. C. S. 1983, PASP, 95, 842 
Shen, Z.-Q., Wan, T.-S., Moran, J. M., et al. 1998 AJ, 115, 1357

Small, T. A., Sargent, W. L. W., \& Steidel, C. C. 1997, AJ, 114, 2254

Smith, P. S., Schmidt, G. D., Jannuzi, B. T., \& Elston, R. 1994, ApJ, 426, 535

Snellen, I. A., Schilizzi, R. T., de Bruyn, A. G., \& Miley, G. K. 1998, å, 333,70

Snellen, I. A. G, Schilizzi, R. T., Bremer, M. N., et al. H. J. A. 1999, MNRAS, 307, 149

Spencer, R. E., McDowell, J. C., Charlesworth, M., et al. 1989, MNRAS, 240, 675

Stanghellini, C., O’Dea, C. P., Baum, S. A., et al. 1996, Int. Astron. Union Symp., 175, 67

Stanghellini, C., O’Dea, C. P., Baum, S. A., et al. 1997, A\&A, 325, 943

Stanghellini, C., O’Dea, C. P., Dallacasa, D., et al. 1998, A\&AS, 131, 303

Steppe, H., Paubert, G., Sievers, A., et al. 1993, A\&AS, 102, 611

Steppe, H., Jeyakumar, S., Saikia, D. J., \& Salter, C. J. 1995, A\&AS, 113,409

Stevens, J. A., Litchfield, S. J., Robson, E. I., et al. 1995, MNRAS, 275,1146

Stickel, M., \& Kühr, H. 1993, A\&AS, 101, 521

Stickel, M., Fried, J. W., \& Kuehr, H. 1993, A\&AS, 98, 393

Stickel, M., Rieke, G. H., Kuehr, H., \& Rieke, M. J. 1996, ApJ, 468, 556

Tapia, S., Craine, E. R., Gearhart, M. R., Pacht, E., \& Kraus, J. 1977, ApJS, 215, 71

Taylor, G. B. 2000, ApJ, 533, 95

Taylor, G. B., Ge, J., \& O’Dea, C. P. 1995, AJ, 110, 522,

Teräsranta, H., Valtaoja, E., Haarala, S., et al. 1987, A\&AS, 71, 125

Teräsranta, H., Tornikoski, M., Valtaoja, E., et al. 1992, A\&AS, 94, 121

Teräsranta, H., Tornikoski, M., Mujunen, A., et al. 1998, A\&A, 132, 305

Toffolatti, L., Argüeso Gómez, F., de Zotti, G., et al. 1997, MNRAS, 297,117

Torniainen, I., et al. 2005, A\&A, in preparation

Tornikoski, M., Valtaoja, E., Terasranta, H., et al. 1993, AJ, 105, 1680

Tornikoski, M., Valtaoja, E., Teräsranta, H., et al. 1996, A\&AS, 116, 157
Tornikoski, M., Lainela, M., \& Valtaoja, E. 2000, AJ, 120, 2278

Tornikoski, M., Jussila, I., Johansson, P., Lainela, M., \& Valtaoja, E. 2001, AJ, 121, 1306

Tornikoski, M., Lähteenmäki, A., Lainela, M., \& Valtaoja, E. 2002, ApJ, 579, 136

Tornikoski, M., \& Valtaoja, E. 2005, A\&A, in preparation

Ulvestad, J., Johnston, K., Perley, R., \& Fomalont, E. 1981, AJ, 86, 1010

Ulvestad, J., Johnston, K., Perley, R., \& Fomalont, E. 1982, AJ, 86, 1010

Valtaoja, E., Teräsranta, H., Urpo, S., et al. 1992, 254, 71

Venturi, T., Pearson, T. J., Barthel, P. D., \& Unwin, S. C. 1997, A\&A, 325,484

Verkhodanov, O. V., Trushkin, S. A., Andernach, H., \& Chernenkov V. N. 1997, Astronomical DAta Analysis Software and Systems VI, ed. G. Hunt, \& H. E. Payne, ASP Conf. Ser., 125, 322

Vermeulen, R. C., Ros, E., \& Kellerman, K. I., et al. 2003, A\&A, 401, 112

Veron, P. 1994, A\&A, 283, 802

Veron, P. 1996, A\&A, 310, 381

Veron-Cetty, M. P., \& Veron, P. 1993, ESO Scientific report, Garching: European Southern Observatory (ESO), 6th ed.

Veron-Cetty, M. P., \& Veron, P. 1998, ESO Scientific report, Garching: European Southern Observatory (ESO), 18th ed.

Villata, M., Raiteri, C. M., Ghisellini, G., et al. 1997, A\&AS, 121, 119

Visser, A. E., Riley, J. M., Roettgering, H. J. A., \& Waldram, E. M. 1995, A\&AS, 110, 419

Waltman, E. B., Fiedler, R. L., Johnston, K. J., et al. 1991, ApJS, 77, 379

White, R. L., \& Becker, R. H. 1992, ApJS, 79, 331

Wilkinson, P. N., Akujor, C. E., Cornwell, T. J., \& Saikia, D. J. 1991, MNRAS, 248, 86

Wiren, S., Valtaoja, E., Teräsranta, H., \& Kotilainen, J. 1992, AJ, 103, 3

Wills, D., \& Wills, B. J. 1976, ApJS, 31, 143

Wills, B. J., Wills, D., Breger, M., Antonucci, R. R. J., \& Barvainis, R. 1992, ApJ, 398, 454

Wright, A. E., \& Otrupcek, R. 1996, yCat, 8015

Wright, A. E., Wark, R. M., Troup, E., et al. 1991, MNRAS, 251, 330

Zirbel, E. L., \& Baum, S. A. 1998, ApJS, 114, 177 